\title{
A JOINT FUNCTIONAL CALCULUS FOR SECTORIAL OPERATORS WITH COMMUTING RESOLVENTS
}

\author{
FLORENCE LANCIEN, GILLES LANCIEN, and CHRISTIAN LE MERDY
}

[Received 26 June 1996-Revised 14 March 1997 and 12 June 1997]

\section{Introduction}

In this paper we are concerned with $H^{\infty}$ functional calculus in the sense of the construction introduced by McIntosh [23] and developed recently by Cowling, Doust, McIntosh, and Yagi [8]. Given $\theta$ in $(0, \pi]$, we will use the notation $\Sigma_{\theta}=\{z \in \mathbb{C} \backslash\{0\}:|\arg z|<\theta\}$ to denote the open sector of angle $2 \theta$ around the half-line $\mathbb{R}_{+}$. Let $X$ be a complex Banach space. Given a linear operator $A$ on $X$ we denote by $D(A), N(A)$ and $R(A)$ the domain, the kernel and the range of $A$ respectively. We also denote by $\sigma(A)$ the spectrum of $A$ and by $\rho(A)$ the resolvent set of $A$.

Definition 1.1. Let $\omega \in(0, \pi)$ and let $A$ be a linear operator on $X$. We say that $A$ is pseudosectorial of type $\omega$ if $A$ is closed, $D(A)$ is dense in $X$, the spectrum $\sigma(A)$ is included in $\overline{\Sigma_{\omega}}$, and

$$
\forall \theta \in(\omega, \pi), \exists C>0 \text { such that } \forall z \in \mathbb{C} \backslash \overline{\Sigma_{\theta}},\left\|z(A-z)^{-1}\right\| \leqslant C .
$$

If, moreover, $N(A)=\{0\}$ and $R(A)$ is dense, we simply say that $A$ is sectorial of type $\omega$.

The purpose of this paper is the study of a joint $H^{\infty}$ functional calculus for a pair of sectorial operators $A, B$ on $X$ with commuting resolvents, that is, which satisfy

$$
\forall \lambda \in \rho(A), \forall \mu \in \rho(B), \quad(\lambda-A)^{-1}(\mu-B)^{-1}=(\mu-B)^{-1}(\lambda-A)^{-1} .
$$

This joint functional calculus, which was first introduced by Albrecht [1], is a natural two-variable analogue of McIntosh's $H^{\infty}$ functional calculus. Given two sectorial operators $A$ and $B$ which are of types $\omega$ and $\omega^{\prime}$ respectively and satisfy (1.2), and two numbers $\mu \in(\omega, \pi), \mu^{\prime} \in\left(\omega^{\prime}, \pi\right)$, it consists of defining a closed and densely defined operator $F(A, B)$ for any bounded analytic function $F: \Sigma_{\mu} \times \Sigma_{\mu^{\prime}} \rightarrow \mathbb{C}$, in a way that preserves reasonable algebraic and continuity properties. This leads to the notion of bounded $H^{\infty}$ joint functional calculus for a pair of commuting sectorial operators. See $\S 2$ below for precise definitions and basic properties.

The first problem addressed in this paper is the following: what are the Banach spaces $X$ for which $(A, B)$ admits a bounded $H^{\infty}$ joint functional calculus as soon as $A$ and $B$ each admit a bounded $H^{\infty}$ functional calculus? In [1], Albrecht proves

1991 Mathematics Subject Classification: 47A60, 47D06, 46C15. 
that this is the case if $X$ is an $L^{p}$-space, with $1<p<+\infty$. We extend this result to a class of Banach spaces containing, in particular, every Banach space having local unconditional structure and also every quotient of subspaces of $B$-convex Banach lattices.

Later in the paper we apply these results to the study of the sum and the product of two commuting sectorial operators $A$ and $B$. As usual, the operators $A+B$ and $A B$ are here understood with their natural domains:

$$
\begin{gathered}
D(A+B)=D(A) \cap D(B), \\
D(A B)=\{x \in D(B): B x \in D(A)\} .
\end{gathered}
$$

We recall that the assumption (1.2) ensures that $D(A+B)$ and $D(A B)$ are dense and that $A+B$ and $A B$ are closable operators (see, for example, [30]).

Assume now that the sum of the types of $A$ and $B$ is strictly less than $\pi$. Then a classical problem, which goes back at least to [9], is to determine conditions under which $A+B$ is closed. This problem turns out to be strongly related to joint functional calculus. Indeed the closedness of $A+B$ is implied by the boundedness of $A(A+B)^{-1}$, which is equivalent to boundedness of $F(A, B)$, where $F\left(z, z^{\prime}\right)=z\left(z+z^{\prime}\right)^{-1}$ (see Proposition 2.7 below). A remarkable theorem of Dore and Venni [11] (completed by Prüss and Sohr [30]) asserts that $A+B$ is closed under the following conditions:

(i) the Banach space $X$ has the UMD property (that is, the Hilbert transform is bounded on $\left.L^{2}(\mathbb{R} ; X)\right)$, and

(ii) $A$ and $B$ each admit bounded imaginary powers and there exist four positive constants $K, K^{\prime}, \mu, \mu^{\prime}$, such that $\mu+\mu^{\prime}<\pi$ and $\left\|A^{i s}\right\| \leqslant K e^{\mu|s|}$, $\left\|B^{i s}\right\| \leqslant K^{\prime} e^{\mu^{\prime}|s|}$ for all $s$ in $\mathbb{R}$.

Furthermore, it is proved in $[\mathbf{1 2}, \mathbf{3 0}]$ that under these conditions, $A+B$ and $\overline{A B}$ actually admit bounded imaginary powers. We will obtain in $\S 4$ the following $H^{\infty}$ functional calculus analogue of the Dore-Venni-Prüss-Sohr theorem. Note however that the geometric assumption on $X$ in Theorem 1.2 is different from the UMD property.

Theorem 1.2. Let $X$ be a Banach space. Assume that $X$ is either a Banach lattice or the quotient of two subspaces of a B-convex Banach lattice (for instance, a quotient of subspaces of $L^{p}$, where $\left.1<p<+\infty\right)$. Let $A$ and $B$ be two sectorial operators on $X$, with commuting resolvents. Assume that $A$ admits a bounded $H^{\infty}\left(\Sigma_{\mu}\right)$ functional calculus, $B$ admits a bounded $H^{\infty}\left(\Sigma_{\mu^{\prime}}\right)$ functional calculus, and $\mu+\mu^{\prime}<\pi$.

Then $A+B$ is a sectorial operator (in particular, $A+B$ is closed) and

(i) for any $\nu>\operatorname{Max}\left\{\mu, \mu^{\prime}\right\}, A+B$ admits a bounded $H^{\infty}\left(\Sigma_{\nu}\right)$ functional calculus,

(ii) for any $\nu>\mu+\mu^{\prime}, \overline{A B}$ admits a bounded $H^{\infty}\left(\Sigma_{\nu}\right)$ functional calculus.

In the last two sections of this paper, we study another variant of McIntosh's functional calculus which also leads to the study of the boundedness of $A(A+B)^{-1}$ (for two commuting operators $A$ and $B$ ) and discuss some connections with the study of maximal regularity (in the $L^{p}$-sense) for the Cauchy problem. 
Let $A$ be a sectorial operator on a $B$-convex Banach lattice $\Lambda$ (for example, $\Lambda$ is an $L^{p}$-space with $\left.1<p<+\infty\right)$ and let $\mathscr{A}$ be its natural extension to $\Lambda(H)$, where $H$ is a Hilbert space. Assume that $\mathscr{A}$ (or equivalently $A$ ) admits a bounded $H^{\infty}\left(\Sigma_{\mu}\right)$ functional calculus. The main result in $\S 5$ says that for any $\nu>\mu$, this functional calculus extends in a natural way to a bounded Banach algebra homomorphism from $H^{\infty}\left(\Sigma_{\nu} ; B(H)\right)$ into $B(\Lambda(H))$.

Let us now recall the definition of maximal regularity, to which we apply this result. Note that with the terminology introduced in Definition 1.1, a linear operator $B$ on a Banach space $X$ is pseudosectorial of type strictly less than $\frac{1}{2} \pi$ if and only if $(-B)$ is the infinitesimal generator of a bounded holomorphic semigroup on $X$; see, for example, [16].

Definition 1.3. Let $T>0$ and $1<p<+\infty$ be two numbers. Let $X$ be a Banach space and let $B$ be a pseudosectorial operator of type strictly less than $\frac{1}{2} \pi$ on $X$. Then $B$ is said to have the maximal regularity property if there exists $C>0$ such that for any $f$ in $L^{p}(0, T ; X)$ there exists a unique function $u$ in $W_{0}^{1, p}(0, T ; X) \cap L^{p}(0, T ; D(B))$ satisfying

$$
u^{\prime}+B u=f \text { on }[0, T) \text { and }\|u\| \leqslant C\|f\| .
$$

It is well known that this property does not depend on $1<p<+\infty$ and $0<T<+\infty$. Moreover, if $X$ is a Hilbert space, then any $B$ as above has the maximal regularity property. This is due to De Simon [10]. It is an open problem to decide whether any pseudosectorial operator of type less than $\frac{1}{2} \pi$ has the maximal regularity property on $L^{p}$ for $1<p \neq 2<+\infty$. However great progress was made by Dore and Venni [11] (see also [30]) by means of their theorem quoted above. Indeed they proved that if $X$ is a UMD Banach space (in particular, if $X=L^{p}$, for $1<p<+\infty$ ), and if $B$ admits bounded imaginary powers which satisfy $\left\|B^{i s}\right\| \leqslant K^{\prime} e^{\mu^{\prime}|s|}$ for some constants $K^{\prime}>0$ and $0<\mu^{\prime}<\frac{1}{2} \pi$, then $B$ has the maximal regularity property.

In the following theorem, which will be proved in $\S 5$, we obtain a sufficient condition for maximal regularity which combines the results of De Simon and Dore and Venni, at least to some extent.

THEOREM 1.4. Let $\left(\Omega_{1}, m_{1}\right)$ be a measure space and let $1<q<+\infty$. Let $H$ be a Hilbert space and $B$ an operator on $L^{q}\left(\Omega_{1}, m_{1}\right)$ with a bounded $H^{\infty}\left(\Sigma_{\mu}\right)$ functional calculus for some $\mu<\frac{1}{2} \pi$. Let $C$ be any pseudosectorial operator of type strictly less than $\frac{1}{2} \pi$ on $H$. Let $\tilde{B}$ and $\tilde{C}$ be the closures, which exist, of $B \otimes I_{H}$ and $I_{L^{q}} \otimes C$ respectively.

Then $\tilde{B}+\tilde{C}$ is a sectorial operator of type strictly less than $\frac{1}{2} \pi$ with the maximal regularity property.

This result can be interpreted as follows. Let $\left(T_{t}\right)_{t \geqslant 0}$ be a bounded holomorphic semigroup on $X=L^{q}\left(\Omega_{1}, m_{1} ; H\right)$. Assume that $\left(T_{t}\right)_{t \geqslant 0}$ is obtained as a tensor product, that is, for any $t \geqslant 0, T_{t}$ is the closure of $U_{t} \otimes V_{t}$ where $\left(U_{t}\right)_{t \geqslant 0}$ and $\left(V_{t}\right)_{t \geqslant 0}$ are two bounded holomorphic semigroups defined on $L^{q}\left(\Omega_{1}, m_{1}\right)$ and $H$ respectively. Then to obtain the maximal regularity property for the negative generator of $\left(T_{t}\right)_{t \geqslant 0}$, it suffices to know that, for some $\mu<\frac{1}{2} \pi$, the negative generator of $\left(U_{t}\right)_{t \geqslant 0}$ has a bounded $H^{\infty}\left(\Sigma_{\mu}\right)$ functional calculus on $L^{q}\left(\Omega_{1}, m_{1}\right)$. 
Acknowledgements. We wish to thank David Albrecht, Edwin Franks and Alan McIntosh who let us know of their recent work on $H^{\infty}$ functional calculus. We are very grateful to Alan McIntosh for fruitful conversations on the material presented in this paper. We also thank Yves Raynaud and Quanhua $\mathrm{Xu}$ for answering several questions on Banach lattices, and the anonymous referee whose numerous suggestions improved the presentation of the paper.

\section{Preliminaries}

First, we introduce the main notation. For $\theta$ in $(0, \pi)$, we denote by $\Gamma_{\theta}$ the oriented contour defined by

$$
\Gamma_{\theta}(t)= \begin{cases}-t e^{i \theta} & \text { if }-\infty<t \leqslant 0 \\ t e^{-i \theta} & \text { if } 0 \leqslant t<+\infty\end{cases}
$$

We will also often use the function $\varphi$ defined on $\mathbb{C} \backslash\{-1\}$ by $\varphi(z)=z /(1+z)^{2}$.

In order to study the boundedness of special operators such as $A(A+B)^{-1}$, it will be useful to use a variant of McIntosh's one-variable $H^{\infty}$ functional calculus. To define it, we replace scalar-valued holomorphic functions by operator-valued holomorphic functions.

Given $\mu \in(0, \pi)$ and a Banach space $E$, we will denote by $H^{\infty}\left(\Sigma_{\mu} ; E\right)$ the Banach space of bounded holomorphic functions $f: \Sigma_{\mu} \rightarrow E$, equipped with the norm

$$
\|f\|=\operatorname{Sup}\left\{\|f(z)\|_{E}: z \in \Sigma_{\mu}\right\} \text {. }
$$

Let $A$ be a sectorial operator of type $\omega(0<\omega<\pi)$ on a complex Banach space $X$. The commutant of $A$, denoted $E_{A}$, is defined to be the closed subalgebra of $B(X)$,

$$
E_{A}=\left\{T \in B(X): T(\lambda-A)^{-1}=(\lambda-A)^{-1} T, \lambda \in \rho(A)\right\} .
$$

It is easy to check that a bounded operator $T$ on $X$ belongs to $E_{A}$ as soon as it commutes with $(\lambda-A)^{-1}$ for one element $\lambda$ in $\rho(A)$.

Let $\mu>\omega$ and let $E$ be a closed subalgebra of $E_{A}$. We now consider $H_{0}^{\infty}\left(\Sigma_{\mu} ; E\right)$, the space of all $f$ in $H^{\infty}\left(\Sigma_{\mu} ; E\right)$ for which there exists $s>0$ such that $\varphi^{-s} f$ belongs to $H^{\infty}\left(\Sigma_{\mu} ; E\right)$. Let $\theta$ be in $(\omega, \mu)$. Then for any $f$ in $H_{0}^{\infty}\left(\Sigma_{\mu} ; E\right)$, we can define

$$
u_{A}(f)=\frac{1}{2 \pi i} \int_{\Gamma_{\theta}} f(\lambda)(\lambda-A)^{-1} d \lambda
$$

which is an integral converging in $B(X)$ and whose value does not depend on the choice of $\theta$.

Now observe that the operator $u_{A}(\varphi)=\varphi(A)=A(I+A)^{-2}$ is one-to-one with range $D(A) \cap R(A)$, which is a dense subspace of $X$ since $A$ is a sectorial operator; see [30]. Let $f$ be any function in $H^{\infty}\left(\Sigma_{\mu} ; E\right)$. The product function $f \varphi$ belongs to $H_{0}^{\infty}\left(\Sigma_{\mu} ; E\right)$. Hence it makes sense to define

$$
u_{A}(f)=\varphi(A)^{-1} u_{A}(f \varphi)
$$

with domain given, via (1.4), by

$$
D\left(u_{A}(f)\right)=\left\{x \in X: u_{A}(f \varphi) x \in D(A) \cap R(A)\right\} .
$$

From the inclusion $E \subset E_{A}$, it follows easily that $u_{A}$ is an algebra homomorphism from $H_{0}^{\infty}\left(\Sigma_{\mu} ; E\right)$ into $B(X)$. This implies that for any $f$ in $H^{\infty}\left(\Sigma_{\mu} ; E\right), D\left(u_{A}(f)\right)$ 
contains $R(\varphi(A))=D(A) \cap R(A)$ and is therefore dense. Since $\varphi(A)$ is bounded, $u_{A}(f)$ is a closed operator. Then, by the closed graph theorem, $u_{A}(f)$ is bounded if and only if $D\left(u_{A}(f)\right)=X$.

We say that $A$ admits a bounded $H^{\infty}\left(\Sigma_{\mu} ; E\right)$ functional calculus if $u_{A}(f)$ is bounded for any $f \in H^{\infty}\left(\Sigma_{\mu} ; E\right)$. These definitions coincide with those in [8, 23] in the case when $E=\operatorname{Span}\left\{I_{X}\right\}$. As in that case, the fundamental proposition is as follows.

Proposition 2.1. The following assertions are equivalent:

(i) A admits a bounded $H^{\infty}\left(\Sigma_{\mu}\right.$;E) functional calculus;

(ii) there exists $C>0$ such that for all $f \in H_{0}^{\infty}\left(\Sigma_{\mu} ; E\right),\left\|u_{A}(f)\right\| \leqslant C\|f\|_{H^{\infty}\left(\Sigma_{\mu} ; E\right)}$;

(iii) there exists $C>0$ such that for all $f \in H^{\infty}\left(\Sigma_{\mu} ; E\right), u_{A}(f) \in B(X)$ and $\left\|u_{A}(f)\right\| \leqslant C\|f\|_{H^{\infty}\left(\Sigma_{\mu} ; E\right)}$.

In this case, $u_{A}$ is a (bounded) homomorphism on $H^{\infty}\left(\Sigma_{\mu} ; E\right)$.

Of course when $E=\operatorname{Span}\left\{I_{X}\right\}$, we merely speak of $H^{\infty}\left(\Sigma_{\mu}\right)$ functional calculus instead of $H^{\infty}\left(\Sigma_{\mu} ; E\right)$ functional calculus.

If $A$ is pseudosectorial and if (ii) is satisfied, we will say that $A$ admits a bounded $H_{0}^{\infty}\left(\Sigma_{\mu} ; E\right)$ functional calculus.

We now turn to the definition of the joint $H^{\infty}$ functional calculus. The following construction was first introduced by Albrecht [1]. It is entirely parallel to McIntosh's one-variable construction.

Given $\mu, \mu^{\prime} \in(0, \pi)$, we denote by $H^{\infty}\left(\Sigma_{\mu} \times \Sigma_{\mu^{\prime}}\right)$ the Banach algebra of all bounded holomorphic scalar-valued functions on $\Sigma_{\mu} \times \Sigma_{\mu^{\prime}}$ and we define $\Phi$ by

Then we define

$$
\Phi\left(z, z^{\prime}\right)=\varphi(z) \varphi\left(z^{\prime}\right)=\frac{z z^{\prime}}{(1+z)^{2}\left(1+z^{\prime}\right)^{2}} .
$$

$$
H_{0}^{\infty}\left(\Sigma_{\mu} \times \Sigma_{\mu^{\prime}}\right)=\left\{F \in H^{\infty}\left(\Sigma_{\mu} \times \Sigma_{\mu^{\prime}}\right): \exists s>0, \Phi^{-s} F \in H^{\infty}\left(\Sigma_{\mu} \times \Sigma_{\mu^{\prime}}\right)\right\} .
$$

Let $A$ and $B$ be two sectorial operators on a Banach space $X$, of types $\omega$ and $\omega^{\prime}$ respectively, with commuting resolvents. For $\mu>\omega, \mu^{\prime}>\omega^{\prime}$, and $F \in$ $H_{0}^{\infty}\left(\Sigma_{\mu} \times \Sigma_{\mu^{\prime}}\right)$ one defines

$$
F(A, B)=-\frac{1}{4 \pi^{2}} \int_{\Gamma_{\theta} \times \Gamma_{\theta^{\prime}}} F\left(\lambda, \lambda^{\prime}\right)(\lambda-A)^{-1}\left(\lambda^{\prime}-B\right)^{-1} d \lambda d \lambda^{\prime}
$$

with $\left(\theta, \theta^{\prime}\right) \in(\omega, \mu) \times\left(\omega^{\prime}, \mu^{\prime}\right)$. This integral converges in $B(X)$ and does not depend on the choice of $\left(\theta, \theta^{\prime}\right)$. To define $F(A, B)$ for general $F$ we proceed as in the one-variable case. For any $F$ in $H^{\infty}\left(\Sigma_{\mu} \times \Sigma_{\mu^{\prime}}\right)$, the function $F \Phi$ belongs to $H_{0}^{\infty}\left(\Sigma_{\mu} \times \Sigma_{\mu^{\prime}}\right)$; hence we may set

$$
F(A, B)=\Phi(A, B)^{-1}(F \Phi)(A, B) .
$$

This makes sense since $\Phi(A, B)$ is one-to-one.

As in the first situation, the calculus associated with $(A, B)$ on $\Sigma_{\mu} \times \Sigma_{\mu^{\prime}}$ by the formula (2.3) defines an algebra homomorphism from $H_{0}^{\infty}\left(\Sigma_{\mu} \times \Sigma_{\mu^{\prime}}\right)$ into $B(X)$. To go further, we need the auxiliary functions $\varphi_{n}$ and $\Phi_{n}$ defined for all $n \geqslant 1$ by

$$
\varphi_{n}(z)=\frac{n^{2} z}{(n+z)(1+n z)} \quad \text { and } \quad \Phi_{n}\left(z, z^{\prime}\right)=\varphi_{n}(z) \varphi_{n}\left(z^{\prime}\right) .
$$


Lemma 2.2. For any $k \geqslant 1$, we let $R_{k}=R\left(\Phi^{k}(A, B)\right)$. Then

(i) for all $k \geqslant 1$ and all $x \in X, \lim _{n \rightarrow \infty} \Phi_{n}^{k}(A, B) x=x$,

(ii) for all $k \geqslant 1$ and all $n \geqslant 1, R\left(\Phi_{n}^{k}(A, B)\right)=R_{k}$,

(iii) for all $k \geqslant 1, R_{k}$ is dense in $X$,

(iv) for all $k \geqslant 1, \Phi^{k}(A, B)$ is a bounded, one-to-one operator with dense range.

Proof. It is well known that when $A$ and $B$ are sectorial, then, for any $x$ in $X$, $\lim _{n \rightarrow \infty} \varphi_{n}(A) x=\lim _{n \rightarrow \infty} \varphi_{n}(B) x=x$ (see, for example, [29, § 8.1]). Moreover the sequences $\left(\varphi_{n}(A)\right)_{n \geqslant 1},\left(\varphi_{n}(B)\right)_{n \geqslant 1}$ are bounded, whence the result (i) follows. The rest of the lemma is then clear.

Let $F \in H^{\infty}\left(\Sigma_{\mu} \times \Sigma_{\mu^{\prime}}\right)$ and let $F(A, B)$ be defined by (2.4). It is a closed operator for the domain defined by $D(F(A, B))=\left\{x \in X:(F \Phi)(A, B) \in R_{1}\right\}$. By the homomorphism property, $D(F(A, B))$ contains $R_{1}$; hence it is dense in $X$ by Lemma 2.2. Consequently, $F(A, B)$ is bounded if and only if $D(F(A, B))=X$. Moreover the definitions (2.3) and (2.4) are consistent for $F$ in $H_{0}^{\infty}\left(\Sigma_{\mu} \times \Sigma_{\mu^{\prime}}\right)$.

We will now prove, for completeness, a useful convergence lemma, which is a variant of the usual convergence lemma due to McIntosh [23].

LEMMA 2.3. Let $\left(F_{n}\right)_{n \geqslant 1}$ be a bounded sequence in $H_{0}^{\infty}\left(\Sigma_{\mu} \times \Sigma_{\mu^{\prime}}\right)$ and $F$ be in $H^{\infty}\left(\Sigma_{\mu} \times \Sigma_{\mu^{\prime}}\right)$ such that $\left(F_{n}\right)_{n \geqslant 1}$ converges pointwise to $F$ on $\Sigma_{\mu} \times \Sigma_{\mu^{\prime}}$. Then:

(i) for all $x \in R_{1}, \lim _{n \rightarrow \infty}\left\|F_{n}(A, B) x-F(A, B) x\right\|=0$;

(ii) if, moreover, $\sup _{n \geqslant 1}\left\|F_{n}(A, B)\right\|$ is finite, then $F(A, B)$ is bounded.

Proof. Let us denote $G=F \Phi$ and $G_{n}=F_{n} \Phi$. For $x$ in $R_{1}$, there is $y \in X$ such that $x=\Phi(A, B) y$. So

$$
\begin{aligned}
& \left\|F_{n}(A, B) x-F(A, B) x\right\| \\
& \quad=\left\|G_{n}(A, B) y-G(A, B) y\right\| \\
& \quad=\left\|\frac{1}{4 \pi^{2}} \int_{\Gamma_{\theta} \times \Gamma_{\theta^{\prime}}}\left(G_{n}\left(\lambda, \lambda^{\prime}\right)-G\left(\lambda, \lambda^{\prime}\right)\right)(\lambda-A)^{-1}\left(\lambda^{\prime}-B\right)^{-1} y d \lambda d \lambda^{\prime}\right\|,
\end{aligned}
$$

for some $\left(\theta, \theta^{\prime}\right) \in(\omega, \mu) \times\left(\omega^{\prime}, \mu^{\prime}\right)$. Now, (i) follows from Lebesgue's dominated convergence theorem and then (ii) is clear.

We say that $(A, B)$ admits a bounded $H^{\infty}\left(\Sigma_{\mu} \times \Sigma_{\mu^{\prime}}\right)$ joint functional calculus if $F(A, B)$ is a bounded operator for any $F$ in $H^{\infty}\left(\Sigma_{\mu} \times \Sigma_{\mu^{\prime}}\right)$. By combining Lemma 2.3 and the closed graph theorem as in [23], one can transfer Proposition 2.1 to the two-variable setting.

Proposition 2.4. The following assertions are equivalent:

(i) $(A, B)$ admits a bounded $H^{\infty}\left(\Sigma_{\mu} \times \Sigma_{\mu^{\prime}}\right)$ joint functional calculus;

(ii) there exists $C>0$ such that for all $F \in H_{0}^{\infty}\left(\Sigma_{\mu} \times \Sigma_{\mu^{\prime}}\right),\|F(A, B)\| \leqslant$ $C\|F\|_{H^{\infty}\left(\Sigma_{\mu} \times \Sigma_{\mu^{\prime}}\right)}$

(iii) there exists $C>0$ such that for all $F \in H^{\infty}\left(\Sigma_{\mu} \times \Sigma_{\mu^{\prime}}\right), F(A, B) \in B(X)$ and $\|F(A, B)\| \leqslant C\|F\|_{H^{\infty}\left(\Sigma_{\mu} \times \Sigma_{\mu^{\prime}}\right)}$. 
In this case, the map $F \mapsto F(A, B)$ is a (bounded) homomorphism on $H^{\infty}\left(\Sigma_{\mu} \times \Sigma_{\mu^{\prime}}\right)$.

Next we indicate how one can in a standard way extend this calculus to an even larger class than $H^{\infty}\left(\Sigma_{\mu} \times \Sigma_{\mu^{\prime}}\right)$, namely the class of all analytic functions of polynomial growth on $\Sigma_{\mu} \times \Sigma_{\mu^{\prime}}$. Let $P$ be an analytic function on $\Sigma_{\mu} \times \Sigma_{\mu^{\prime}}$; we say that $P$ is of polynomial growth if there is $k$ in $\mathbb{N}$ such that $P \Phi^{k} \in H_{0}^{\infty}\left(\Sigma_{\mu} \times \Sigma_{\mu^{\prime}}\right)$. For such a function $P$, we define $P(A, B)=\Phi(A, B)^{-k}\left(P \Phi^{k}\right)(A, B)$. By the homomorphism property, this definition does not depend on $k$ such that $P \Phi^{k} \in H_{0}^{\infty}\left(\Sigma_{\mu} \times \Sigma_{\mu^{\prime}}\right)$ and by Lemma 2.2 we immediately obtain the following.

Proposition 2.5. Let $A$ and $B$ be two sectorial operators on $X$ with respective types $\omega$ and $\omega^{\prime}$ and with commuting resolvents. Let $\left(\mu, \mu^{\prime}\right) \in(\omega, \pi) \times\left(\omega^{\prime}, \pi\right)$ and $P$ be an analytic function of polynomial growth on $\Sigma_{\mu} \times \Sigma_{\mu^{\prime}}$. Then $P(A, B)$ is a closed operator whose domain is dense in $X$.

We will now state two propositions that will be very useful for our applications to maximal regularity problems.

Proposition 2.6. Let $A$ be a sectorial operator of type $\omega$ and $B$ a pseudosectorial operator of type $\omega^{\prime}$, whose resolvents commute. Assume $\omega+\omega^{\prime}<\pi$. Then $R(A+B)$ is dense in $X$ and $\overline{A+B}$ is injective.

Now let $\omega<\mu<\pi-\omega^{\prime}$. If we define $f$ in $H^{\infty}\left(\Sigma_{\mu} ; E_{A}\right)$ by $f(z)=z(z+B)^{-1}$, then the following assertions are equivalent:

(i) $u_{A}(f) \in B(X)$;

(ii) $A+B$ is a closed operator and

$$
\exists C>0 \text { such that } \forall x \in D(A) \cap D(B),\|A x\| \leqslant C\|A x+B x\| .
$$

Proof. Let $\omega<\mu<\pi-\omega^{\prime}$. Consider $\sigma$ and $\tau$ in $H_{0}^{\infty}\left(\Sigma_{\mu} ; E_{A}\right)$ defined by

$$
\sigma(z)=(z+B)(1+B)^{-1} \varphi^{2}(z) \quad \text { and } \quad \tau(z)=(z+B)^{-1} \varphi^{2}(z) .
$$

The homomorphism property yields $(1+B)^{-1} \varphi^{4}(A)=u_{A}(\sigma) u_{A}(\tau)$. Since $u_{A}(\sigma)=$ $(A+B)(1+B)^{-1} \varphi^{2}(A)$, we deduce that $R\left((1+B)^{-1} \varphi^{4}(A)\right) \subset R(A+B)$. Consider now $x=(1+B)^{-1} y$ in $D(B)$. Since $(1+B)^{-1} \varphi_{n}^{4}(A) y \rightarrow x$, we see that $R\left((1+B)^{-1} \varphi^{4}(A)\right)$ is dense in $D(B)$ and therefore dense in $X$. Consequently $R(A+B)$ is dense in $X$.

Now let $x$ be in $N(\overline{A+B})$. There exists a sequence $\left(x_{n}\right)$ in $D(A) \cap D(B)$ such that $x_{n} \rightarrow x$ and $(A+B) x_{n} \rightarrow 0$. Passing to the limit in the equality $(1+B)^{-1} \varphi^{4}(A) x_{n}=u_{A}(\sigma) u_{A}(\tau) x_{n}$, one obtains $(1+B)^{-1} \varphi^{4}(A) x=0$, and therefore $x=0$. This proves the first part of the proposition.

Turning to the second part, we will now show that

$$
\forall x \in D(A) \cap D(B), \quad u_{A}(f \varphi)(A+B) x=\varphi(A) A x .
$$


So let $x \in D(A) \cap D(B)$; then given $\theta$ in $(\omega, \mu)$, we have

$$
\begin{aligned}
u_{A}\left(f \varphi^{2}\right)(A+B) x & =\frac{1}{2 \pi i} \int_{\Gamma_{\theta}} \varphi^{2}(\lambda) \lambda(\lambda+B)^{-1}(\lambda-A)^{-1}(A+B) x d \lambda \quad \text { (by (2.1)) } \\
& =\frac{1}{2 \pi i} \int_{\Gamma_{\theta}} \lambda \varphi^{2}(\lambda)(\lambda-A)^{-1} x d \lambda-\frac{1}{2 \pi i} \int_{\Gamma_{\theta}} \lambda \varphi^{2}(\lambda)(\lambda+B)^{-1} x d \lambda \\
& =A \varphi^{2}(A) x \quad \text { (by Cauchy's theorem). }
\end{aligned}
$$

Since $x \in D(A)$, we obtain $\varphi(A) u_{A}(f \varphi)(A+B) x=\varphi^{2}(A) A x$. Then (2.6) follows from the injectivity of $\varphi(A)$.

Let us show that (i) implies (ii). We assume that $u_{A}(f)$ is bounded. Then it follows again from the injectivity of $\varphi(A)$ that for any $x \in D(A) \cap D(B)$, $u_{A}(f)(A+B) x=A x$. This clearly implies (2.5) and the closedness of $A+B$ can then be deduced in the usual way.

Assume now that the assertion (ii) is satisfied. From (2.6) it follows that

$$
\forall y \in \varphi(A)(R(A+B)), \quad\left\|u_{A}(f) y\right\| \leqslant C\|y\| .
$$

So we can conclude our proof by showing that $\varphi(A)(R(A+B))$ is dense in $X$. Since $\overline{R(A+B)}=X$, it is enough to prove that $\varphi(A)(R(A+B))$ is dense in $R(A+B)$. This relies on the following standard arguments: let $x=(A+B) y$ in $R(A+B)$; then $x_{n}=\varphi_{n}(A) x \rightarrow x$ and $x_{n} \in \varphi(A)(R(A+B))$.

With essentially the same proof, we obtain the following result.

Proposition 2.7. Let $A$ and $B$ be two sectorial operators with commuting resolvents and respective types $\omega$ and $\omega^{\prime}$. Assume $\omega+\omega^{\prime}<\pi$ and let $\omega<\mu<\pi-\omega^{\prime}$ and $\omega^{\prime}<\mu^{\prime}<\pi-\mu$. Define $F$ in $H^{\infty}\left(\Sigma_{\mu} \times \Sigma_{\mu^{\prime}}\right)$ by $F\left(z, z^{\prime}\right)=z /\left(z+z^{\prime}\right)$ for any $\left(z, z^{\prime}\right) \in \Sigma_{\mu} \times \Sigma_{\mu^{\prime}}$. Then the following assertions are equivalent:

(i) $F(A, B) \in B(X)$;

(ii) $A+B$ is a closed operator and

$$
\exists C>0 \text { such that } \forall x \in D(A) \cap D(B),\|A x\| \leqslant C\|A x+B x\| .
$$

We will continue this section by fixing some notation and recalling some definitions related to the general geometric properties of the Banach spaces that we will consider in this paper.

Definition 2.8. Let $X$ be a Banach space and denote by $r_{n}(t)=$ $\operatorname{sign}\left(\sin \left(2^{n} \pi t\right)\right)$, for $n \geqslant 1$, the Rademacher functions on the interval $I=[0,1]$.

(i) We say that $X$ is of type $p$, for $1<p \leqslant 2$, if there is a constant $C$ such that for all finite subsets $\left\{x_{1}, \ldots, x_{n}\right\}$ of $X$,

$$
\left\|\sum_{i=1}^{n} r_{i} x_{i}\right\|_{L^{1}(I ; X)} \leqslant C\left(\sum_{i=1}^{n}\left\|x_{i}\right\|^{p}\right)^{1 / p} .
$$

(ii) We say that $X$ is of cotype $q$, for $2 \leqslant q<+\infty$, if there is a constant $C$ 
such that for all finite subsets $\left\{x_{1}, \ldots, x_{n}\right\}$ of $X$,

$$
\left(\sum_{i=1}^{n}\left\|x_{i}\right\|^{q}\right)^{1 / q} \leqslant C\left\|\sum_{i=1}^{n} r_{i} x_{i}\right\|_{L^{1}(I ; X)} .
$$

(iii) We say that a Banach space $X$ is $B$-convex if it is of type $p$, for some $p>1$.

Finally, we recall an interpolation theorem of Carleson. For convenience, we will transpose to $\Sigma_{\pi / 2}$ the statement that can be found in [15] for the upper half plane.

THEOREM 2.9 (Carleson). Let $\left(z_{j}\right)_{j} \geqslant 1$ be a sequence in $\Sigma_{\pi / 2}$. Then the following conditions are equivalent:

(i) there is $\delta>0$ such that

$$
\forall k \geqslant 1, \quad \prod_{j \neq k}\left|\frac{z_{k}-z_{j}}{z_{k}+\bar{z}_{j}}\right| \geqslant \delta ;
$$

(ii) there exist a sequence $\left(f_{j}\right)_{j \geqslant 1}$ in $H^{\infty}\left(\Sigma_{\pi / 2}\right)$ and a constant $M>0$ such that

$$
\begin{gathered}
\forall j \geqslant 1, f_{j}\left(z_{j}\right)=1 ; \quad \forall k \neq j, f_{j}\left(z_{k}\right)=0 ; \\
\text { and } \quad \forall z \in \Sigma_{\pi / 2}, \quad \sum_{j \geqslant 1}\left|f_{j}(z)\right| \leqslant M .
\end{gathered}
$$

Such a sequence $\left(z_{j}\right)_{j \geqslant 1}$ is called an interpolating sequence in $\Sigma_{\pi / 2}$. Indeed, let $Y$ be a Banach space and $\left(y_{j}\right)_{j} \geqslant 1$ a bounded sequence in $Y$. Then the function $f$ defined on $\Sigma_{\pi / 2}$ by $f(z)=\sum_{j \geqslant 1} f_{j}(z) y_{j}$ satisfies the following properties:

$$
f \in H^{\infty}\left(\Sigma_{\pi / 2} ; Y\right), \quad\|f\|_{H^{\infty}\left(\Sigma_{\pi / 2} ; Y\right)} \leqslant M \sup _{j \geqslant 1}\left\|y_{j}\right\| \quad \text { and } \quad \forall j \geqslant 1, f\left(z_{j}\right)=y_{j} .
$$

\section{On Banach spaces with the joint calculus property}

In this section we describe large classes of Banach spaces having the following property.

Definition 3.1. We say that a Banach space $X$ has the joint calculus property if whenever $A$ and $B$ are sectorial operators on $X$ with commuting resolvents, which are of types $\omega$ and $\omega^{\prime}$ respectively and admit a bounded $H^{\infty}\left(\Sigma_{\mu}\right)$ or $H^{\infty}\left(\Sigma_{\mu^{\prime}}\right)$ respectively, functional calculus (where $0<\omega<\mu$ and $0<\omega^{\prime}<\mu^{\prime}$ ), then $(A, B)$ admits a bounded $H^{\infty}\left(\Sigma_{\beta} \times \Sigma_{\beta^{\prime}}\right)$ joint functional calculus for any $\left(\beta, \beta^{\prime}\right)$ in $(\mu, \pi) \times\left(\mu^{\prime}, \pi\right)$.

Let us first mention that there exist Banach spaces without the joint calculus property. For example, it can be deduced from [24] that the Banach space of all compact operators on $\ell_{2}$ does not have this property. As we mentioned in our introduction, Albrecht [1] proved that $L^{p}$-spaces, with $1<p<\infty$, have the joint calculus property. Recently, Franks and McIntosh [14] obtained a simpler proof of Albrecht's theorem based on a decomposition result for analytic functions. Since this decomposition will be an important tool in this section, we now recall it precisely. 
Franks and McIntosh [14, §3] proved the following: for any $\left(\mu, \mu^{\prime}\right)$ in $(0, \pi) \times(0, \pi)$ and any $\left(\beta, \beta^{\prime}\right)$ in $(\mu, \pi) \times\left(\mu^{\prime}, \pi\right)$, there are a constant $C>0$ and sequences $\left(\psi_{i}\right)_{i \geqslant 0},\left(\tilde{\psi}_{i}\right)_{i \geqslant 0}$ in $H_{0}^{\infty}\left(\Sigma_{\mu}\right)$, and $\left(\varphi_{j}\right)_{j \geqslant 0},\left(\tilde{\varphi}_{j}\right)_{j \geqslant 0}$ in $H_{0}^{\infty}\left(\Sigma_{\mu^{\prime}}\right)$ such that

$$
\begin{aligned}
\forall p>0, \quad \sup _{\Sigma_{\mu}} \sum_{i \geqslant 0}\left|\psi_{i}\right|^{p}, \sup _{\Sigma_{\mu}} \sum_{i \geqslant 0}\left|\tilde{\psi}_{i}\right|^{p}, \sup _{\Sigma_{\mu^{\prime}}} & \sum_{j \geqslant 0}\left|\varphi_{j}\right|^{p} \text {, and } \\
& \sup _{\Sigma_{\mu^{\prime}}} \sum_{j \geqslant 0}\left|\tilde{\varphi}_{j}\right|^{p} \text { are finite, }
\end{aligned}
$$

and for all $h \in H^{\infty}\left(\Sigma_{\beta} \times \Sigma_{\beta^{\prime}}\right)$, there exists $\left(\alpha_{i, j}\right) \in \mathbb{C}^{\mathbb{N}^{2}}$ such that for all $\zeta=$ $\left(\zeta_{1}, \zeta_{2}\right) \in \Sigma_{\mu} \times \Sigma_{\mu^{\prime}}$

$$
\begin{array}{r}
h(\zeta)=\sum_{i, j \geqslant 0} \alpha_{i, j} \psi_{i}\left(\zeta_{1}\right) \tilde{\psi}_{i}\left(\zeta_{1}\right) \varphi_{j}\left(\zeta_{2}\right) \tilde{\varphi}_{j}\left(\zeta_{2}\right) \\
\text { with } \sup _{i, j \geqslant 0}\left|\alpha_{i, j}\right| \leqslant C\|h\|_{H^{\infty}\left(\Sigma_{\beta} \times \Sigma_{\beta^{\prime}}\right)}
\end{array}
$$

A complete reference for the notions of geometry of Banach spaces that we will use can be found in $[\mathbf{2 0}, \mathbf{2 1}, \mathbf{2 8}$. However, we will recall a few definitions. We say that a Banach space $X$ has a local unconditional structure (l.u.st.) if there is a constant $\lambda$ such that for any finite-dimensional subspace $F$ of $X$, there is a space $E$ with an unconditional basis $\left(e_{n}\right)$ and there are operators $A \in B(F, E)$ and $B \in B(E, X)$ such that $B A$ is the canonical embedding of $F$ into $X$ and $\|A\|\|B\| K \leqslant \lambda$, where $K$ is the unconditional basis constant of $\left(e_{n}\right)$. Any Banach lattice has this property and it is even known (see [13]) that a Banach space has l.u.st. if and only if its bidual is isomorphic to a complemented subspace of a Banach lattice. We will also consider the following class of Banach spaces, introduced by Pisier [25]: a Banach space $X$ has property $(\alpha)$ if there exists a constant $C>0$ such that the inequality

$$
\begin{aligned}
& \left\|\sum_{1 \leqslant i, j \leqslant n} \alpha_{i, j}\left(r_{i} \otimes r_{j}\right) x_{i, j}\right\|_{L^{2}(I \times I ; X)} \\
& \quad \leqslant C \sup _{1 \leqslant i, j \leqslant n}\left|\alpha_{i, j}\right|\left\|_{1 \leqslant i, j \leqslant n}\left(r_{i} \otimes r_{j}\right) x_{i, j}\right\|_{L^{2}(I \times I ; X)}
\end{aligned}
$$

is satisfied for every integer $n$ and every choice of $\left(\alpha_{i, j}\right)$ in $\mathbb{C}^{n^{2}}$ and $\left(x_{i, j}\right)$ in $X^{n^{2}}$ (where $\left(r_{n}\right)$ denotes the sequence of Rademacher functions on $I=[0,1]$ ).

The main results of this section are the following.

THEOREM 3.2. Every Banach space with a local unconditional structure has the joint calculus property.

Theorem 3.3. Let $X$ be a Banach space such that $X$ or $X^{*}$ has property $(\alpha)$. Then $X$ has the joint calculus property.

Before proceeding with the proofs of these theorems we need to introduce a technical intermediate property: we say that a Banach space $X$ satisfies property (A) if there is a constant $C>0$ such that, for any $n$ in $\mathbb{N},\left(\alpha_{i, j}\right)$ in $\mathbb{C}^{n^{2}},\left(x_{i, j}\right)$ in 
$X^{n^{2}}$ and $\left(x_{i, j}^{*}\right)$ in $\left(X^{*}\right)^{n^{2}}$,

$$
\begin{aligned}
\left|\sum_{1 \leqslant i, j \leqslant n} \alpha_{i, j}\left\langle x_{i, j}, x_{i, j}^{*}\right\rangle\right| \leqslant & C \sup _{1 \leqslant i, j \leqslant n}\left|\alpha_{i, j}\right| \\
& \times\left\|_{1 \leqslant i, j \leqslant n}\left(r_{i} \otimes r_{j}\right) x_{i, j}\right\|_{L^{2}(I \times I ; X)} \\
& \times\left\|_{1 \leqslant i, j \leqslant n}\left(r_{i} \otimes r_{j}\right) x_{i, j}^{*}\right\|_{L^{2}\left(I \times I ; X^{*}\right)}
\end{aligned}
$$

Then the proofs of both Theorems 3.2 and 3.3 rely on the following.

Proposition 3.4. If a Banach space X satisfies property (A), then it has the joint calculus property.

Proof. We keep the notation introduced in Definition 3.1 and apply the analytic decomposition given by (3.1) and (3.2).

To any $h$ in $H^{\infty}\left(\Sigma_{\beta} \times \Sigma_{\beta^{\prime}}\right)$, we associate the partial sums of its decomposition given by (3.2), namely, $h_{n}=\sum_{1 \leqslant i, j \leqslant n} \alpha_{i, j}\left(\psi_{i} \tilde{\psi}_{i} \otimes \varphi_{j} \tilde{\varphi}_{j}\right)$ for all $n \geqslant 1$. By Lemma 2.3 , in order to show that $(A, B)$ admits a bounded $H^{\infty}\left(\Sigma_{\beta} \times \Sigma_{\beta^{\prime}}\right)$ functional calculus, it is enough to find a constant $K>0$ such that

$$
\forall h \in H^{\infty}\left(\Sigma_{\beta} \times \Sigma_{\beta^{\prime}}\right), \quad \forall n \geqslant 1, \quad\left\|h_{n}(A, B)\right\| \leqslant K\|h\|_{H^{\infty}\left(\Sigma_{\beta} \times \Sigma_{\beta^{\prime}}\right)} .
$$

So, let $x \in X$ and $x^{*} \in X^{*}$. Then

$$
\begin{aligned}
\left|\left\langle h_{n}(A, B) x, x^{*}\right\rangle\right| & =\left|\sum_{1 \leqslant i, j \leqslant n} \alpha_{i, j}\left\langle\psi_{i}(A) \varphi_{j}(B) x, \tilde{\psi}_{i}(A)^{*} \tilde{\varphi}_{j}(B)^{*} x^{*}\right\rangle\right| \\
\leqslant & C\|h\|_{H^{\infty}\left(\Sigma_{\beta} \times \Sigma_{\beta^{\prime}}\right)}\left\|\sum_{1 \leqslant i, j \leqslant n}\left(r_{i} \otimes r_{j}\right) \psi_{i}(A) \boldsymbol{\varphi}_{j}(B) x\right\|_{L^{2}(I \times I ; X)} \\
& \times\left\|\sum_{1 \leqslant i, j \leqslant n}\left(r_{i} \otimes r_{j}\right) \tilde{\psi}_{i}(A)^{*} \tilde{\varphi}_{j}(B)^{*} x^{*}\right\|_{L^{2}\left(I \times I ; X^{*}\right)}
\end{aligned}
$$

by (3.2) and property (A) for $X$. Now, fix $(s, t) \in I \times I$. Using the fact that $A$ and $B$ admit a bounded $H^{\infty}$ functional calculus on $\Sigma_{\mu}$ and $\Sigma_{\mu^{\prime}}$, respectively, we have

$$
\begin{aligned}
& \left\|\sum_{1 \leqslant i, j \leqslant n} r_{i}(s) r_{j}(t) \psi_{i}(A) \varphi_{j}(B) x\right\|_{X} \\
& \leqslant\left\|\sum_{1 \leqslant i \leqslant n} r_{i}(s) \psi_{i}(A)\right\|\left\|_{B(X)}\right\| \sum_{1 \leqslant j \leqslant n} r_{j}(t) \varphi_{j}(B)\left\|_{B(X)}\right\| x \| \\
& \leqslant K_{1}\left\|\sum_{1 \leqslant i \leqslant n} r_{i}(s) \psi_{i}\right\|_{H^{\infty}\left(\Sigma_{\mu}\right)}\left\|\sum_{1 \leqslant j \leqslant n} r_{j}(t) \varphi_{j}\right\|_{H^{\infty}\left(\Sigma_{\mu^{\prime}}\right)}\|x\| \\
& \leqslant K_{2}\|x\| \text { by }(3.1) .
\end{aligned}
$$


Therefore

$$
\left\|\sum_{1 \leqslant i, j \leqslant n}\left(r_{i} \otimes r_{j}\right) \psi_{i}(A) \varphi_{j}(B) x\right\|_{L^{2}(I \times I ; X)} \leqslant K_{2}\|x\|
$$

Similarly

$$
\left\|\sum_{1 \leqslant i, j \leqslant n}\left(r_{i} \otimes r_{j}\right) \tilde{\psi}_{i}(A)^{*} \tilde{\varphi}_{j}(B)^{*} x^{*}\right\|_{L^{2}\left(I \times I ; X^{*}\right)} \leqslant K_{3}\left\|x^{*}\right\|,
$$

whence (3.3) follows.

It follows rather easily from Goldstine's Lemma that if a Banach space $X$ has property (A), so does its dual. Since the converse implication is obvious, we can state the following.

Lemma 3.5. Let $X$ be a Banach space. Then $X$ has property (A) if and only if its dual $X^{*}$ has property (A).

Proof of Theorem 3.2. Applying Proposition 3.4, we wish to show that any Banach space with a local unconditional structure satisfies property (A). We know that a Banach space has 1.u.st. if and only if its bidual is isomorphic to a complemented subspace of a Banach lattice [13]. Moreover, it is clear that if $X$ satisfies property (A), so does any complemented subspace of $X$. By Lemma 3.5, it is therefore enough to show that any Banach lattice satisfies property (A).

Let $X$ be a Banach lattice. Then for any $\left(\alpha_{i, j}\right) \in \mathbb{C}^{n^{2}},\left(x_{i, j}\right) \in X^{n^{2}}$ and $\left(x_{i, j}^{*}\right) \in\left(X^{*}\right)^{n^{2}}$,

$$
\left|\sum \alpha_{i, j}\left\langle x_{i, j}, x_{i, j}^{*}\right\rangle\right| \leqslant \sup \left|\alpha_{i, j}\right|\left\|\left(\sum\left|x_{i, j}\right|^{2}\right)^{1 / 2}\right\|_{X}\left\|\left(\sum\left|x_{i, j}^{*}\right|^{2}\right)^{1 / 2}\right\|_{X^{*}} .
$$

Let us say a word about the meaning of this inequality. If the reader is not a specialist in Banach lattices, a good way to get some intuition is to imagine $X$ (and then $X^{*}$ ) as a function lattice on a measure space $\Omega$. Then, the quantity $\left(\sum\left|x_{i, j}(\omega)\right|^{2}\right)^{1 / 2}$ makes sense for almost every $\omega$ in $\Omega$ and turns out to define an element of $X$. The above inequality is thus a consequence of the pointwise application of the Cauchy-Schwarz inequality. A meaning can also be given to $\left(\sum\left|x_{i, j}\right|^{2}\right)^{1 / 2}$ in general Banach lattices (see [21] for complete details) and the previous inequality holds true as well.

The following two-variable version of Khintchine's inequality is well known:

$$
\exists C \text { such that } \forall\left(a_{i, j}\right)_{1 \leqslant i, j \leqslant n} \in \mathbb{C}^{n^{2}}, \quad\left(\sum\left|a_{i, j}\right|^{2}\right)^{1 / 2} \leqslant C\left\|\sum a_{i, j} r_{i} \otimes r_{j}\right\|_{L^{1}(I \times I)} \text {. }
$$

This can be viewed as a particular case of Kahane's inequality (see, for example, [21, p. 74]). The latter inequality implies that, for any $\left(x_{i, j}\right)$ in $X^{n^{2}}$,

$$
\left\|\left(\sum\left|x_{i, j}\right|^{2}\right)^{1 / 2}\right\|_{X} \leqslant C\left\|\int_{I^{2}}\left|\sum r_{i}(t) r_{j}(s) x_{i, j}\right| d s d t\right\|_{X}
$$

whence

$$
\left\|\left(\sum\left|x_{i, j}\right|^{2}\right)^{1 / 2}\right\|_{X} \leqslant C\left\|\sum\left(r_{i} \otimes r_{j}\right) x_{i, j}\right\|_{L^{2}\left(I^{2} ; X\right)} .
$$

The inequalities (3.4) and (3.5) yield the desired property (A) for $X$. 
Proof of Theorem 3.3. Assume, for instance, that $X$ has property $(\alpha)$ and let us show property (A). With the above notation we have

$$
\begin{aligned}
& \left|\sum_{1 \leqslant i, j \leqslant n} \alpha_{i, j}\left\langle x_{i, j}, x_{i, j}^{*}\right\rangle\right| \\
& =\left|\int_{I \times I}\left\langle\sum_{i, j} \alpha_{i, j} r_{i}(t) r_{j}(s) x_{i, j}, \sum_{k, \ell} r_{k}(t) r_{\ell}(s) x_{k, \ell}^{*}\right\rangle d s d t\right| \\
& \leqslant\left.\left\|\sum_{i, j} \alpha_{i, j}\left(r_{i} \otimes r_{j}\right) x_{i, j}\right\|_{L^{2}(I \times I ; X)}\left\|\sum_{i, j}\left(r_{i} \otimes r_{j}\right) x_{i, j}^{*}\right\|\right|_{L^{2}\left(I \times I ; X^{*}\right)} \\
& \leqslant C \sup _{i, j}\left|\alpha_{i, j}\right|\left\|\sum_{i, j}\left(r_{i} \otimes r_{j}\right) x_{i, j}\right\|_{L^{2}(I \times I ; X)}\left\|\sum_{i, j}\left(r_{i} \otimes r_{j}\right) x_{i, j}^{*}\right\|_{L^{2}\left(I \times I ; X^{*}\right)}
\end{aligned}
$$

by the $(\alpha)$ property of $X$. When $X^{*}$ has property $(\alpha)$, we just have to modify this proof in an obvious manner.

In [25], Pisier exhibited several classes of Banach spaces with property $(\alpha)$. From his results and Theorem 3.3, we deduce the next result.

COROLlary 3.6. Let E be a Banach space with l.u.st. (or merely the GordonLewis property).

(i) If $E$ has a finite cotype, then every subspace of $E$ has the joint calculus property.

(ii) If $E^{*}$ has a finite cotype, then every quotient of $E$ has the joint calculus property.

(iii) If $E$ and $E^{*}$ have finite cotype, then every quotient of subspaces of $E$ has the joint calculus property.

We refer the reader to [28] for information on the Gordon-Lewis property. From (i) it follows that every subspace of $L^{1}$ has the joint calculus property and from (iii) that every quotient of subspaces of a $B$-convex Banach lattice (or equivalently a Banach lattice with a finite cotype and such that its dual has a finite cotype) has the joint calculus property. We mention for completeness that Bourgain [3] constructed a $B$-convex Banach lattice which is not a UMD space. In order to describe the field of application of our results, let us finally notice that for any measure space $(\Omega, \Sigma, \mu)$ and any $1 \leqslant p<+\infty$, if a Banach space $X$ enjoys property $(\alpha)$, so does $L^{p}(\Omega ; X)$.

We will now study the joint calculus property for a few Banach spaces that are typical for their lack of unconditional structure.

Let $\mathbb{T}=\{z \in \mathbb{C}:|z|=1\}$ and $D=\{z \in \mathbb{C}:|z|<1\}$. We let $L^{p}=L^{p}(\mathbb{T})$ for $1 \leqslant p \leqslant \infty$, where $\mathbb{T}$ is equipped with its Haar measure. Let $H^{p}=$ $\left\{f \in L^{p}: \forall n<0, \int_{\mathbb{T}} f(z) z^{n} d z=0\right\}$ be the usual Hardy spaces over $D$. Lastly we denote by $A(D)$ the disk algebra which is the space of continuous functions on $\mathbb{}$ belonging to $H^{\infty}$. We denote by $P$ the quotient mapping from $L^{1}$ onto $L^{1} / H^{1}$. We can now state together our positive results about these spaces. 
THEOREM 3.7. The spaces $L^{1} / H^{1}, H^{\infty}$, and $A(D)$ enjoy the joint calculus property.

Proof. We will show that these spaces satisfy (A). We start with $L^{1} / H^{1}$. In [4] Bourgain proved that $L^{1} / H^{1}$ is a Grothendieck space of cotype 2 or, equivalently (see [28]), that it satisfies the following lifting property. There is a constant $C>0$ such that, for any subset $\left\{x_{1}, \ldots, x_{n}\right\}$ of $L^{1} / H^{1}$, there is a subset $\left\{y_{1}, \ldots, y_{n}\right\}$ of $L^{1}$ such that

$$
\forall i \in\{1, \ldots, n\}, \quad P y_{i}=x_{i} \quad \text { and }\left\|\sum_{i=1}^{n} r_{i} y_{i}\right\|_{L^{2}\left(I ; L^{1}\right)} \leqslant C\left\|\sum_{i=1}^{n} r_{i} x_{i}\right\|_{L^{2}\left(I ; L^{1} / H^{1}\right)} .
$$

A thorough examination of the proof given in [6] (see also [28, Chapter 6]) yields the following lifting property with two variables. There is a constant $C>0$ such that, for any subset $\left\{x_{i, j}\right\}_{1 \leqslant i, j \leqslant n}$ of $L^{1} / H^{1}$, there is a subset $\left\{y_{i, j}\right\}_{1 \leqslant i, j \leqslant n}$ of $L^{1}$ such that

$$
\forall 1 \leqslant i, j \leqslant n, \quad P y_{i, j}=x_{i, j}
$$

and

$$
\left\|\sum_{1 \leqslant i, j \leqslant n}\left(r_{i} \otimes r_{j}\right) y_{i, j}\right\|_{L^{2}\left(I \times I ; L^{1}\right)} \leqslant C\left\|_{1 \leqslant i, j \leqslant n}\left(r_{i} \otimes r_{j}\right) x_{i, j}\right\|_{L^{2}\left(I \times I ; L^{1} / H^{1}\right)} .
$$

Since $L^{1}$ satisfies property (A), it follows clearly from (3.6) that $L^{1} / H^{1}$ also enjoys property (A) and therefore the joint calculus property by Proposition 3.4.

Notice now that $H^{\infty}$ is isometric to $\left(L^{1} / H^{1}\right)^{*}$. So $H^{\infty}$ has property (A) by Lemma 3.5. Recall finally that the dual of $A(D)$ is isometric to the $\ell_{1}$-sum $L^{1} / H^{1} \oplus_{1} L_{s}^{1}$, where $L_{s}^{1}$ denotes the space of all singular measures on $\mathbb{T}$. We have already seen that $L^{1} / H^{1}$ has property (A). Since it is a Banach lattice, so does $L_{S}^{1}$. Therefore $A(D)^{*}$, and thus $A(D)$, have property (A).

REMARK 3.8. In fact it is possible to deduce from (3.6) that $L^{1} / H^{1}$ has property $(\alpha)$. Moreover, using similar arguments as in the proof of Theorem 3.7, one obtains that for any reflexive subspace $R$ of an $L^{1}$-space $L^{1}(\Omega)$, the quotient space $L^{1}(\Omega) / R$ has the joint calculus property. More generally, if $X$ is a Banach space with property (A) and if $Y \subset X$ is a subspace, then $X / Y$ has property (A) (and thus the joint calculus property) as soon as $Y$ is $B$-convex (see $[\mathbf{2 7}, \mathbf{2 8}]$ ).

We will conclude this section with a counter-example to bounded joint functional calculus on Schatten spaces. Let $S_{\infty}$ be the Banach space of all compact operators on $\ell_{2}$. For $1 \leqslant p<\infty$, the Schatten space $S_{p}$ is defined as the space of all $T$ in $S_{\infty}$ such that $|T|^{p}$ has a finite trace. It can be equipped with the norm $\alpha_{p}(T)=\left(\operatorname{tr}|T|^{p}\right)^{1 / p}$, for which it is a Banach space.

THEOREM 3.9. For all $1 \leqslant p \leqslant \infty$, with $p \neq 2$, the space $S_{p}$ fails to have the joint calculus property.

Proof. Let $1 \leqslant p \leqslant \infty$ with $p \neq 2$. We denote by $\left(e_{j}\right)_{j \geqslant 1}$ the canonical basis of $\ell_{2}$. For any $i, j \geqslant 1$, we let $E_{i, j}$ be the rank 1 operator on $\ell_{2}$ defined by $E_{i, j} x=\left\langle x, e_{j}\right\rangle e_{i}$ for every $x$ in $\ell_{2}$. Let $K_{00}$ be the linear span of the operators $E_{i, j}$. 
We consider it as equipped with the $S_{p}$-norm. For every family $a=\left(a_{i, j}\right)_{i, j \geqslant 1}$ of complex numbers, we denote by $M_{a}: K_{00} \rightarrow K_{00}$ the so-called Schur multiplier defined by $M_{a}\left(E_{i, j}\right)=a_{i, j} E_{i, j}$. When $M_{a}$ is bounded, we say that it is a bounded Schur multiplier on $S_{p}$.

We identify elements of $S_{p}$ with their infinite matrices with respect to the canonical basis of $\ell_{2}$. Let $\left(\alpha_{i}\right)_{i=1}^{\infty}$ and $\left(\beta_{i}\right)_{i=1}^{\infty}$ be two sequences of positive numbers. Let $\Delta_{A}$ and $\Delta_{B}$ be the two diagonal matrices

$$
\Delta_{A}=\left(\begin{array}{cccc}
\alpha_{1} & & & 0 \\
& \ddots & & \\
& & \alpha_{i} & \\
0 & & & \ddots
\end{array}\right) \text { and } \Delta_{B}=\left(\begin{array}{cccc}
\beta_{1} & & & 0 \\
& \ddots & & \\
& & \beta_{i} & \\
0 & & & \ddots
\end{array}\right) .
$$

Then, one can define two sectorial operators $A$ and $B$ of type 0 (that is, of type $\omega$ for any $\omega>0$ ) on $S_{p}$ as follows: let $D(A)=\left\{T \in S_{p}: \Delta_{A} T \in S_{p}\right\}$ and for $T \in D(A), A(T)=\Delta_{A} T$. Similarly, $D(B)=\left\{T \in S_{p}: T \Delta_{B} \in S_{p}\right\}$ and for $T \in D(B)$, $B(T)=T \Delta_{B}$. For any $\mu$ in $(0, \pi), A$ and $B$ admit a bounded $H^{\infty}\left(\Sigma_{\mu}\right)$ functional calculus. Indeed, for any $f$ in $H^{\infty}\left(\Sigma_{\mu}\right)$, and any $T$ in $S_{p}$, we have

$$
f(A)(T)=f\left(\Delta_{A}\right) T \text { and } f(B)(T)=T f\left(\Delta_{B}\right)
$$

where

$$
f\left(\Delta_{A}\right)=\left(\begin{array}{cccc}
f\left(\alpha_{1}\right) & & & 0 \\
& \ddots & & \\
& & f\left(\alpha_{i}\right) & \\
0 & & & \ddots
\end{array}\right) \text { and } f\left(\Delta_{B}\right)=\left(\begin{array}{cccc}
f\left(\beta_{1}\right) & & & 0 \\
& \ddots & & \\
& & f\left(\beta_{i}\right) & \\
0 & & & \ddots
\end{array}\right) \text {. }
$$

Now let $\mu$ and $\mu^{\prime}$ be in $(0, \pi)$ and $F \in H^{\infty}\left(\Sigma_{\mu} \times \Sigma_{\mu^{\prime}}\right)$. Then for every $i, j \geqslant 1, E_{i, j}$ belongs to $D(F(A, B))$ and $F(A, B)\left(E_{i, j}\right)=F\left(\alpha_{i}, \beta_{j}\right) E_{i, j}$. We deduce that

$$
F(A, B) \text { is bounded } \Longleftrightarrow M_{\left(F\left(\alpha_{i}, \beta_{j}\right)\right)} \text { is a bounded Schur multiplier on } S_{p} \text {. }
$$

We now fix $\left(\alpha_{i}\right)_{i \geqslant 1}=\left(\beta_{i}\right)_{i \geqslant 1}=\left(2^{i}\right)_{i \geqslant 1}$ and assume that $(A, B)$ admits a bounded $H^{\infty}\left(\Sigma_{\pi} \times \Sigma_{\pi}\right)$ functional calculus. It can be easily checked that $\left(2^{i}\right)_{i \geqslant 1}$ is an interpolating sequence in $\Sigma_{\pi}$ (that is, $\left(\sqrt{2^{i}}\right)_{i \geqslant 1}$ satisfies (2.7)). So by Theorem 2.9, there exist $M>0$ and $\left(f_{i}\right)_{i \geqslant 1} \subset H^{\infty}\left(\Sigma_{\pi}\right)$ such that

$$
\forall i \geqslant 1, f_{i}\left(2^{i}\right)=1, \quad \forall k \neq i, f_{i}\left(2^{k}\right)=0, \quad \text { and } \quad \forall z \in \Sigma_{\pi}, \quad \sum_{i \geqslant 1}\left|f_{i}(z)\right| \leqslant M .
$$

Let $a=\left(a_{i, j}\right)_{i, j \geqslant 1}$ be any bounded family of complex numbers. We may define $F_{a}$ in $H^{\infty}\left(\Sigma_{\pi} \times \Sigma_{\pi}\right)$ by $F_{a}\left(z, z^{\prime}\right)=\sum_{i, j \geqslant 1} a_{i, j} f_{i}(z) f_{j}\left(z^{\prime}\right)$. Clearly $F_{a}\left(2^{i}, 2^{j}\right)=a_{i, j}$ for every $i, j \geqslant 1$. Hence, by (3.8), $M_{a}$ is a bounded Schur multiplier on $S_{p}$. But the fact that this holds for all bounded $a$ is false, as can be seen in [28, proof of Corollary 8.20].

Remark 3.10. McIntosh and Yagi used the operators considered above in [24] and showed that $A+B$ is not closed in $S_{\infty}$. On the other hand, if $1<p<\infty, S_{p}$ is a UMD space (see [5]) and therefore, the theorem of Dore and Venni ensures that $A+B$ is closed. The Schatten spaces $S_{p}$, for $1<p<\infty$ and $p \neq 2$, provide, to our knowledge, the first examples of UMD spaces that fail the joint calculus property. 


\section{Functional calculus for analytic functions of polynomial growth}

Throughout this section we consider two sectorial operators $A$ and $B$ on a Banach space $X$ with respective types $\omega$ and $\omega^{\prime}$ and with commuting resolvents. We also consider $P$, an analytic function of polynomial growth on $\Sigma_{\mu} \times \Sigma_{\mu^{\prime}}$, where $\left(\mu, \mu^{\prime}\right) \in(\omega, \pi) \times\left(\omega^{\prime}, \pi\right)$. Our main purpose will be to study the functional calculus associated with the closed densely defined operator $P(A, B)$ (see Proposition 2.5), when $(A, B)$ admits a bounded $H^{\infty}$ joint functional calculus. Our general result is the following.

THEOREM 4.1. In the above setting, assume moreover that $(A, B)$ admits a bounded $H^{\infty}\left(\Sigma_{\mu} \times \Sigma_{\mu^{\prime}}\right)$ joint functional calculus and $P\left(\Sigma_{\mu} \times \Sigma_{\mu^{\prime}}\right) \subset \overline{\Sigma_{\eta}}$ for some $\eta \in(0, \pi)$. Then:

(i) $P(A, B)$ is pseudosectorial of type $\eta$;

(ii) if we assume moreover that $P(A, B)$ is one-to-one and has a dense range, then $P(A, B)$ is sectorial of type $\eta$ and admits a bounded $H^{\infty}\left(\Sigma_{\nu}\right)$ functional calculus for any $\nu \in(\eta, \pi)$; furthermore, we have the following composition property:

$$
\forall f \in H^{\infty}\left(\Sigma_{\nu}\right), \quad f(P(A, B))=(f \circ P)(A, B) .
$$

Proof. Let $k \in \mathbb{N}$ such that $P \Phi^{k} \in H_{0}^{\infty}\left(\Sigma_{\mu} \times \Sigma_{\mu^{\prime}}\right)$. Let us first notice that for any $n \geqslant 1, \Phi_{n}^{k} \in H_{0}^{\infty}\left(\Sigma_{\mu} \times \Sigma_{\mu^{\prime}}\right)$ and satisfies

$$
\forall y \in X, \quad P(A, B) \Phi_{n}^{k}(A, B) y=\left(P \Phi_{n}^{k}\right)(A, B) y .
$$

Let us consider $\lambda \in \mathbb{C} \backslash \bar{\Sigma}_{\eta}$. For $\left(z, z^{\prime}\right) \in \Sigma_{\mu} \times \Sigma_{\mu^{\prime}}$ we set $F_{\lambda}\left(z, z^{\prime}\right)=1 /\left(\lambda-P\left(z, z^{\prime}\right)\right)$. Clearly $F_{\lambda} \in H^{\infty}\left(\Sigma_{\mu} \times \Sigma_{\mu^{\prime}}\right)$. Since $(A, B)$ admits a bounded $H^{\infty}\left(\Sigma_{\mu} \times \Sigma_{\mu^{\prime}}\right)$ functional calculus, $F_{\lambda}(A, B) \in B(X)$. Observe that $\left(\lambda \Phi_{n}^{k}-P \Phi_{n}^{k}\right) F_{\lambda}=\Phi_{n}^{k}$ on $\Sigma_{\mu} \times \Sigma_{\mu^{\prime}}$. So, using the homomorphism property and the previous remark, we obtain

$$
\forall x \in X, \quad(\lambda-P(A, B)) \Phi_{n}^{k}(A, B) F_{\lambda}(A, B) x=\Phi_{n}^{k}(A, B) x .
$$

Then as $n$ tends to $\infty$, we obtain, by point (i) of Lemma 2.2 and by the closedness of $P(A, B)$,

$$
\forall x \in X, \quad F_{\lambda}(A, B) x \in D(P(A, B)) \quad \text { and } \quad(\lambda-P(A, B)) F_{\lambda}(A, B) x=x .
$$

On the other hand, we have $F_{\lambda}(A, B)(\lambda-P(A, B)) \Phi_{n}^{k}(A, B)=\Phi_{n}^{k}(A, B)$, which yields, for all $x \in R_{k}, F_{\lambda}(A, B)(\lambda-P(A, B)) x=x$. Therefore, by the closedness of $P(A, B)$ and the boundedness of $F_{\lambda}(A, B)$,

$$
\forall x \in D(P(A, B)), \quad F_{\lambda}(A, B)(\lambda-P(A, B)) x=x .
$$

The identities (4.1) and (4.2) assert that $\lambda \in \rho(P(A, B))$ and that

$$
(\lambda-P(A, B))^{-1}=F_{\lambda}(A, B) .
$$

For $\lambda \in \mathbb{C} \backslash \bar{\Sigma}_{\eta}$, we put $G_{\lambda}=\lambda F_{\lambda}$. Obviously $G_{\lambda}(A, B)=\lambda(\lambda-P(A, B))^{-1}$. Now, for any $\nu \in(\eta, \pi),\left\{G_{\lambda}: \lambda \in \mathbb{C} \backslash \bar{\Sigma}_{\nu}\right\}$ is a bounded subset of $H^{\infty}\left(\Sigma_{\mu} \times \Sigma_{\mu^{\prime}}\right)$. Since $(A, B)$ admits a bounded $H^{\infty}\left(\Sigma_{\mu} \times \Sigma_{\mu^{\prime}}\right)$ functional calculus, $\left\{G_{\lambda}(A, B): \lambda \in \mathbb{C} \backslash \bar{\Sigma}_{\nu}\right\}$ is a bounded subset of $B(X)$. This concludes point (i).

In order to prove (ii), we will show that the composition property is satisfied by any $f$ in $H_{0}^{\infty}\left(\Sigma_{\nu}\right)$. Then the full statement will clearly follow. Note that we do not need to assume that $P(A, B)$ is one-to-one and has dense range in the following computation. 
Let $f$ be in $H_{0}^{\infty}\left(\Sigma_{\nu}\right)$, and set $F=f \circ P$ in $H^{\infty}\left(\Sigma_{\mu} \times \Sigma_{\mu^{\prime}}\right)$. We fix $\theta \in(\omega, \mu)$, $\theta^{\prime} \in\left(\omega^{\prime}, \mu^{\prime}\right)$ and $\theta^{\prime \prime} \in(\eta, \nu)$. By $(2.3)$,

$$
F(A, B) \varphi(A) \varphi(B)=\frac{1}{(2 \pi i)^{2}} \int_{\Gamma_{\theta}} \int_{\Gamma_{\theta^{\prime}}} \varphi(z) \varphi\left(z^{\prime}\right) f\left(P\left(z, z^{\prime}\right)\right)(z-A)^{-1}\left(z^{\prime}-B\right)^{-1} d z d z^{\prime} .
$$

By Cauchy's formula we have, for any $\lambda_{0} \in \bar{\Sigma}_{\eta}$,

$$
f\left(\lambda_{0}\right)=\frac{1}{2 \pi i} \int_{\Gamma_{\theta^{\prime \prime}}} \frac{f(\lambda)}{\lambda-\lambda_{0}} d \lambda
$$

Therefore

$$
\begin{aligned}
F(A, B) & \varphi(A) \varphi(B) \\
= & \frac{1}{(2 \pi i)^{3}} \int_{\Gamma_{\theta}} \int_{\Gamma_{\theta^{\prime}}} \int_{\Gamma_{\theta^{\prime \prime}}} \varphi(z) \varphi\left(z^{\prime}\right) f(\lambda) F_{\lambda}\left(z, z^{\prime}\right)(z-A)^{-1}\left(z^{\prime}-B\right)^{-1} d z d z^{\prime} d \lambda \\
= & \frac{1}{(2 \pi i)} \int_{\Gamma_{\theta^{\prime \prime}}} f(\lambda)(\lambda-P(A, B))^{-1} \varphi(A) \varphi(B) d \lambda \quad \text { (by (4.3)) } \\
= & f(P(A, B)) \varphi(A) \varphi(B) .
\end{aligned}
$$

Since $F(A, B)$ and $f(P(A, B))$ are bounded and since $\varphi(A) \varphi(B)$ has a dense range, we obtain $F(A, B)=f(P(A, B))$.

In order to apply statement (ii) of Theorem 4.1, we need to have the following proposition at our disposal.

Proposition 4.2. Under the assumptions of Theorem 4.1, if we assume moreover that $1 / P$ is of polynomial growth on $\Sigma_{\mu} \times \Sigma_{\mu^{\prime}}$, then $P(A, B)$ is injective and has a dense range and therefore admits a bounded $H^{\infty}\left(\Sigma_{\nu}\right)$ functional calculus, for all $\nu>\eta$.

Proof. Let $k$ be a positive integer such that $P \Phi^{k}$ and $\Phi^{k} / P$ belong to $H_{0}^{\infty}\left(\Sigma_{\mu} \times \Sigma_{\mu^{\prime}}\right)$. From the homomorphism property it follows that

$$
\forall x \in D(P(A, B)), \quad\left(\frac{1}{P}\right)(A, B) \Phi^{2 k}(A, B) P(A, B) x=\Phi^{2 k}(A, B) x .
$$

Since $\Phi^{2 k}(A, B)$ is injective, $P(A, B)$ is injective. Similarly,

$$
P(A, B) \Phi^{k}(A, B)\left(\frac{\Phi^{k}}{P}\right)(A, B)=\Phi^{2 k}(A, B) .
$$

Then, it follows from Lemma 2.2 that $R(P(A, B))$ is dense in $X$.

From now until the end of $\S 4$, we will assume that the couple $(A, B)$ introduced at the beginning of this section admits a bounded $H^{\infty}\left(\Sigma_{\mu} \times \Sigma_{\mu^{\prime}}\right)$ joint functional calculus. We now study in detail a few typical examples of functions of polynomial growth on $\Sigma_{\mu} \times \Sigma_{\mu^{\prime}}$.

ExAmple 1. Let $P_{1}\left(z, z^{\prime}\right)=z+z^{\prime}$ and assume $\mu+\mu^{\prime}<\pi$. Then $P_{1}$ and $1 / P_{1}$ are of polynomial growth on $\Sigma_{\mu} \times \Sigma_{\mu^{\prime}}$ and $P_{1}\left(\Sigma_{\mu} \times \Sigma_{\mu^{\prime}}\right)=\Sigma_{\operatorname{Max}\left\{\mu, \mu^{\prime}\right\}}$. Let us show 
now that $P_{1}(A, B)=A+B$. Since $(A, B)$ admits a bounded $H^{\infty}\left(\Sigma_{\mu} \times \Sigma_{\mu^{\prime}}\right)$ functional calculus, it follows from Proposition 2.7 that $A+B$ is closed. The function $P_{1} \Phi^{2}$ belongs to $H_{0}^{\infty}\left(\Sigma_{\mu} \times \Sigma_{\mu^{\prime}}\right)$ and $\left(P_{1} \Phi^{2}\right)(A, B)$ has an integral representation from which it can easily be shown that $P_{1}(A, B)$ and $A+B$ coincide on $R_{2}=R\left(\Phi^{2}(A, B)\right)$. Now, for any $x$ in $D(A) \cap D(B), \quad\left(x_{n}\right)_{n \geqslant 1}=$ $\left(\Phi_{n}^{2}(A, B) x\right)_{n \geqslant 1}$ is a sequence in $R_{2}$ such that $x_{n} \rightarrow x, A x_{n} \rightarrow A x, B x_{n} \rightarrow B x$ (see Lemma 2.2). Similarly, for any $y$ in $D\left(P_{1}(A, B)\right),\left(y_{n}\right)_{n \geqslant 1}=\left(\Phi_{n}^{2}(A, B) y\right)_{n \geqslant 1}$ is included in $R_{2}, y_{n} \rightarrow y$ and $P_{1}(A, B) y_{n} \rightarrow P_{1}(A, B) y$. Thus the closedness of $A+B$ and $P_{1}(A, B)$ implies that they are equal.

ExAmple 2. Let $P_{2}\left(z, z^{\prime}\right)=z z^{\prime}$ and assume $\mu+\mu^{\prime}<\pi$. Again $P_{2}$ and $1 / P_{2}$ are of polynomial growth on $\Sigma_{\mu} \times \Sigma_{\mu^{\prime}}$ and $P_{2}\left(\Sigma_{\mu} \times \Sigma_{\mu^{\prime}}\right)=\Sigma_{\mu+\mu^{\prime}}$. Using the integral representation of $\left(P_{2} \Phi^{2}\right)(A, B)=P_{2}(A, B) \Phi^{2}(A, B)$, one can easily check that $P_{2}(A, B)$ and $A B$ coincide on $R_{2}$. Then we can use the sequence $\left(\Phi_{n}^{2}(A, B)\right)$ as in Example 1 and it therefore follows from the closedness of $P_{2}(A, B)$ that $P_{2}(A, B)=\overline{A B}$.

If we combine Examples 1 and 2, Theorem 4.1 and Proposition 4.2 we immediately obtain the following.

Corollary 4.3. If $(A, B)$ admits a bounded $H^{\infty}\left(\Sigma_{\mu} \times \Sigma_{\mu^{\prime}}\right)$ joint functional calculus, with $\mu+\mu^{\prime}<\pi$, then:

(i) $A+B$ is a sectorial operator of type $\operatorname{Max}\left\{\mu, \mu^{\prime}\right\}$;

(ii) for all $\nu>\operatorname{Max}\left\{\mu, \mu^{\prime}\right\}, A+B$ admits a bounded $H^{\infty}\left(\Sigma_{\nu}\right)$ functional calculus;

(iii) $\overline{A B}$ is a sectorial operator of type $\mu+\mu^{\prime}$;

(iv) for all $\nu>\mu+\mu^{\prime}, \overline{A B}$ admits a bounded $H^{\infty}\left(\Sigma_{\nu}\right)$ functional calculus.

Proof of Theorem 1.2. Combine Corollary 4.3, Theorem 3.2 and Corollary 3.6.

\section{The $H^{\infty}\left(\Sigma_{\mu} ; B(H)\right)$ functional calculus on $\Lambda(H)$;} applications to maximal regularity

Throughout this section, $\Lambda$ will denote a $B$-convex Banach lattice, or equivalently, a Banach lattice with a finite cotype, whose dual $\Lambda^{*}$ has a finite cotype. We recall that for $1<p<\infty, L^{p}$-spaces are $B$-convex. We will use the following result of Maurey [22] (see also [21, pp.49-50]). If $\Lambda$ is a Banach lattice with a finite cotype, then there is a constant $K>0$ such that, for every subset $\left\{x_{i}\right\}_{i=1}^{n}$ of $\Lambda$,

$$
K^{-1}\left\|\left(\sum_{i=1}^{n}\left|x_{i}\right|^{2}\right)^{1 / 2}\right\|_{\Lambda} \leqslant\left\|\sum_{i=1}^{n} r_{i} x_{i}\right\|_{L^{1}(I ; \Lambda)} \leqslant K\left\|\left(\sum_{i=1}^{n}\left|x_{i}\right|^{2}\right)^{1 / 2}\right\|_{\Lambda} .
$$

Since $\Lambda$ is $B$-convex, it is order continuous and therefore $\Lambda$ and $\Lambda^{*}$ can be represented as function lattices on the same measure space $(\Omega, \Sigma, \mu)$ (see [21, 1.a, 1.b] for details). Moreover, the duality is described by

$$
\forall\left(x, x^{*}\right) \in \Lambda \times \Lambda^{*}, \quad\left\langle x, x^{*}\right\rangle=\int_{\Omega} x(\omega) x^{*}(\omega) d \mu(\omega) .
$$


Now let $X$ be a Banach space. We introduce the following classical definition:

$$
\Lambda(X)=\left\{f: \Omega \rightarrow X: f \text { strongly measurable and }\|f(\cdot)\|_{X} \in \Lambda\right\} .
$$

Note that $\Lambda(X)$ equipped with $\|f\|_{\Lambda(X)}=\|\| f(\cdot)\left\|_{X}\right\|_{\Lambda}$ is a Banach space and the $B$-convexity of $\Lambda$ implies that $\Lambda \otimes X$ is dense in $\Lambda(X)$. As a typical example, notice that if $\Lambda=L^{p}(\Omega, \mu)$ for some $p \in(1,+\infty)$, then $\Lambda(X)=L^{p}(\Omega, \mu ; X)$ is the classical Bochner $X$-valued $L^{p}$-space.

For every $S$ in $B(X), I_{\Lambda} \otimes S$ extends to a bounded operator $\overline{I_{\Lambda} \otimes S}$ on $\Lambda(X)$, with $\left\|\overline{I_{\Lambda} \otimes S}\right\|_{B(\Lambda(X))}=\|S\|_{B(X)}$. This allows us to consider $B(X)$ as a closed subalgebra of $B(\Lambda(X))$. Now let $A$ be a sectorial operator on $\Lambda$. Assume that $A \otimes I_{X}$, defined on $D(A) \otimes X$, is closable in $\Lambda(X)$ and that its closure $\mathscr{A}$ is also sectorial. Then $B(X)$ is actually a subalgebra of $E_{\mathscr{A}}$; thus we have

$$
B(X) \subset E_{\mathscr{A}} \subset B(\Lambda(X)) .
$$

It then makes sense to study the functional calculus $u_{\mathscr{A}}$ (see (2.1), (2.2)) on the Banach algebra $H^{\infty}\left(\Sigma_{\mu} ; B(X)\right)$. It will be shown in $\S 6$ that this functional calculus is not always bounded when $A$ has a bounded $H^{\infty}$ functional calculus. The purpose of this section is to establish positive results in the Hilbert space case and to apply them to maximal regularity. The following introductory proposition deals with the extension to $\Lambda(H)$, where $H$ is a Hilbert space, of a bounded or of a sectorial operator on $\Lambda$.

Proposition 5.1. Let $H$ be a Hilbert space and $0<\omega<\pi$.

(i) There exists a constant $C>0$ such that for every $T$ in $B(\Lambda), T \otimes I_{H}$ is bounded on $\Lambda \otimes H$ and satisfies $\left\|T \otimes I_{H}\right\| \leqslant C\|T\|$. Moreover, if $T$ is injective then its extension $\mathscr{T}=\overline{T \otimes I_{H}}$ to $\Lambda(H)$ is injective.

(ii) If $A$ is a sectorial operator of type $\omega$ on $\Lambda$, then $A \otimes I_{H}$, defined on $D(A) \otimes H$, is a closable operator, whose closure $\mathscr{A}$ is sectorial of type $\omega$ on $\Lambda(H)$. If moreover $A$ admits a bounded $H^{\infty}\left(\Sigma_{\mu}\right)$ functional calculus, so does $\mathscr{A}$.

Proof. We start with the proof of (i). Let $T \in B(\Lambda)$ and let $y$ be in $\Lambda \otimes H$. Then $y=\sum_{i=1}^{n} x_{i} \otimes e_{i}$, where $\left\{x_{i}\right\}_{i=1}^{n} \subset \Lambda$ and $\left(e_{i}\right)_{i=1}^{n}$ is an orthonormal system. We have $\left(T \otimes I_{H}\right) y=\sum_{i=1}^{n} T x_{i} \otimes e_{i}$. Hence to obtain $\left\|T \otimes I_{H}\right\| \leqslant C\|T\|$ it suffices to have

$$
\left\|\left(\sum_{i=1}^{n}\left|T x_{i}\right|^{2}\right)^{1 / 2}\right\|_{\Lambda} \leqslant C\|T\|\left\|\left(\sum_{i=1}^{n}\left|x_{i}\right|^{2}\right)^{1 / 2}\right\|_{\Lambda} .
$$

Such an inequality is actually true for any Banach lattice $\Lambda$ by a result of Krivine [17] (see also [21, p.93]). Note however that under our assumption of $B$-convexity, it can be proved directly as a consequence of (5.1). Indeed, we clearly have

$$
\left\|\sum_{i=1}^{n} r_{i} T x_{i}\right\|_{L^{1}(I ; \Lambda)} \leqslant\|T\|\left\|\sum_{i=1}^{n} r_{i} x_{i}\right\|_{L^{1}(I ; \Lambda)} .
$$

For any $h \in H$ and any $y \in \Lambda(H)$, we may define the element of $\Lambda$, $\tilde{h}(y)=\langle h, y(\cdot)\rangle$. Suppose now that $T$ is injective and let $y$ be in $\Lambda(H)$ such that $\mathscr{T} y=0$. Then for any $h \in H, T(\tilde{h}(y))=\tilde{h}(\mathscr{T} y)=0$. Hence $\tilde{h}(y)=0$, that is, 
$\langle h, y(\omega)\rangle=0$ for almost every $\omega$ in $\Omega$. Since $\Lambda$ is $B$-convex, the essential image of $y$ is separable (see [21, Proposition 1.a.9]) and therefore we obtain $y=0$.

Let us now prove (ii). We give ourselves a sectorial operator $A$ of type $\omega$ on $\Lambda$. Let us consider a sequence $\left(y_{n}\right)_{n=1}^{\infty}$ in $D(A) \otimes H$ such that $y_{n} \rightarrow 0$ and $\left(A \otimes I_{H}\right) y_{n} \rightarrow y$ in $\Lambda(H)$. By point (i) proved above, we can set

$$
\mathscr{R}=\overline{(I+A)^{-1} \otimes I_{H}} \text { in } B(\Lambda(H)) .
$$

We let $z=\mathscr{R} y$. Clearly, $\mathscr{R}\left(A \otimes I_{H}\right) y_{n}=\left(\left(I-(I+A)^{-1}\right) \otimes I_{H}\right) y_{n} \rightarrow z$. Since $\left(I-(I+A)^{-1}\right) \otimes I_{H}$ is bounded, $z=0$. But (i) implies that $\mathscr{R}$ is injective, so $y=0$. The operator $A \otimes I_{H}$ is therefore closable. Moreover $\mathscr{A}=\overline{A \otimes I_{H}}$ clearly has dense range and domain and is injective, for the same reasons as in (i).

In order to conclude this proof we show that $\rho(A)=\rho(\mathscr{A})$ and

$$
\forall \lambda \in \rho(A), \quad(\lambda-\mathscr{A})^{-1}=\overline{(\lambda-A)^{-1} \otimes I_{H}} .
$$

The inclusion $\rho(\mathscr{A}) \subset \rho(A)$ is obvious, so consider $\lambda$ in $\rho(A)$. Then $\lambda-\mathscr{A}$ is injective, so it is enough to prove that

$$
\forall z \in \Lambda(H), \quad y=\left(\overline{(\lambda-A)^{-1} \otimes I_{H}}\right) z \in D(\mathscr{A}) \quad \text { and } \quad(\lambda-\mathscr{A}) y=z .
$$

Let $z \in \Lambda(H)$ and $\left(z_{n}\right)$ be in $\Lambda \otimes H$ such that $z_{n} \rightarrow z$. Let $y_{n}=\left((\lambda-A)^{-1} \otimes I_{H}\right) z_{n}$. Since $(\lambda-A)^{-1} \otimes I_{H}$ is bounded, $\left(y_{n}\right)$ is a Cauchy sequence included in $D(A) \otimes I_{H}$. So $y_{n}$ converges to $y$, for some $y$ in $\Lambda(H)$. Moreover $\mathscr{A} y_{n}=\lambda y_{n}-z_{n} \rightarrow \lambda y-z$. Thus $y \in D(\mathscr{A}),(\lambda-\mathscr{A}) y=z$ and $y=\left(\overline{(\lambda-A)^{-1} \otimes I_{H}}\right) z$.

As an obvious consequence of (5.2) and (i), we see that $\mathscr{A}$ is sectorial of type $\omega$.

Assume finally that $A$ admits a bounded $H^{\infty}\left(\Sigma_{\mu}\right)$ functional calculus. Then it follows from (5.2) and (2.1) that for any $f$ in $H_{0}^{\infty}\left(\Sigma_{\mu}\right), f(\mathscr{A})=\overline{f(A) \otimes I_{H}}$. Thus point (i) and Proposition 2.1 imply that $\mathscr{A}$ admits a bounded $H^{\infty}\left(\Sigma_{\mu}\right)$ functional calculus.

THEOREM 5.2. Let $0<\omega<\mu<\pi$. If $A$ is a sectorial operator of type $\omega$ on $\Lambda$ and admits a bounded $H^{\infty}\left(\Sigma_{\mu}\right)$ functional calculus, then, for any $\nu$ in $(\mu, \pi), \mathscr{A}$ admits a bounded $H^{\infty}\left(\Sigma_{\nu} ; B(H)\right)$ functional calculus.

Let us start with a few preliminary lemmas. The first is an extended version of the quadratic estimates of [8].

Lemma 5.3. Let $x \in \Lambda$ and $\psi \in H_{0}^{\infty}\left(\Sigma_{\mu}\right)$. Then for almost all $\omega$ in $\Omega$, the function $h(\omega): t \mapsto(\psi(t A) x)(\omega)$ belongs to the Hilbert space $H_{0}=$ $L^{2}\left((0, \infty), t^{-1} d t\right)$ and $h$ is an element of $\Lambda\left(H_{0}\right)$.

Moreover, for a given $\psi$ in $H_{0}^{\infty}\left(\Sigma_{\mu}\right)$, there is a constant $C>0$ such that, for any $x$ in $\Lambda,\|h\|_{\Lambda\left(H_{0}\right)} \leqslant C\|x\|_{\Lambda}$.

Proof. Let us fix $\psi$ in $H_{0}^{\infty}\left(\Sigma_{\mu}\right)$. Since $D(A) \cap R(A)$ is dense in $\Lambda$, it suffices to show that this lemma holds for any $x$ in $D(A) \cap R(A)$. So let $x$ belong to $D(A) \cap R(A)$; we can write $x=\varphi(A) y$ with $y$ in $\Lambda$. Then, by Fubini's theorem, the map $f: t \mapsto \psi\left(e^{t} A\right) x$ belongs to $L^{1}(\mathbb{R} ; \Lambda)$ and, for all $t$ in $\mathbb{R}, \hat{f}(t)=\hat{\psi}_{e}(t) A^{i t} x$, where $\psi_{e} \in L^{1}(\mathbb{R})$ is defined by $\psi_{e}(t)=\psi\left(e^{t}\right)$. Then, for almost every $\omega$ in $\Omega$, $f_{\omega}: t \mapsto\left(\psi\left(e^{t} A\right) x\right)(\omega)$ is in $L^{1}(\mathbb{R})$ and, for all $s$ in $\mathbb{R}, \hat{f}_{\omega}(s)=\hat{\psi}_{e}(s)\left(A^{i s} x\right)(\omega)$. Now, 
by Plancherel's theorem, we find that, $\omega$-almost everywhere, $h(\omega) \in H_{0}$ and

$$
\|h(\omega)\|_{H_{0}}=\frac{1}{2 \pi}\left(\int_{-\infty}^{+\infty}\left|\hat{\psi}_{e}(s)\left(A^{i s} x\right)(\omega)\right|^{2} d s\right)^{1 / 2}
$$

Finally, following the proof of Theorem 6.6 in $[\mathbf{8}]$, we find that the right-hand side of (5.3) defines an element of $\Lambda$ whose norm is less than $C\|x\|_{\Lambda}$, where $C$ is a constant independent of $x$.

Next, we generalize Lemma 5.3 to the case of finite sequences in $\Lambda$.

Lemma 5.4. Let $\psi$ be in $H_{0}^{\infty}\left(\Sigma_{\mu}\right)$. There exists a constant $C>0$ such that, for any finite subset $\left\{x_{i}\right\}_{i=1}^{n}$ of $\Lambda$,

$$
\left\|\left(\int_{0}^{\infty} \sum_{i=1}^{n}\left|\psi(t A) x_{i}\right|^{2} \frac{d t}{t}\right)^{1 / 2}\right\|_{\Lambda} \leqslant C\left\|\left(\sum_{i=1}^{n}\left|x_{i}\right|^{2}\right)^{1 / 2}\right\|_{\Lambda}
$$

Proof. By Lemma 5.3 and by the properties of the Banach lattices, both quantities in the inequality (5.4) make sense. So let $\left\{x_{i}\right\}_{i=1}^{n} \subset \Lambda$ and define $h_{i} \in \Lambda\left(H_{0}\right)$ as above by $h_{i}(\omega): t \mapsto\left(\psi(t A) x_{i}\right)(\omega)$. Then, for any fixed $\omega \in \Omega$,

$$
\begin{aligned}
\left(\int_{0}^{\infty} \sum_{i=1}^{n}\left|\psi(t A) x_{i}\right|^{2}(\omega) \frac{d t}{t}\right)^{1 / 2} & =\left(\sum_{i=1}^{n}\left\|h_{i}(\omega)\right\|_{H_{0}}^{2}\right)^{1 / 2} \\
& =\left\|\sum_{i=1}^{n} r_{i} h_{i}(\omega)\right\|_{L^{2}\left(I ; H_{0}\right)} \\
& \leqslant K_{1}\left\|\sum_{i=1}^{n} r_{i} h_{i}(\omega)\right\|_{L^{1}\left(I ; H_{0}\right)}
\end{aligned}
$$

by Kahane's inequality (see, for example, [21, p. 74]). By convexity we then have

$$
\left\|\left(\int_{0}^{\infty} \sum_{i=1}^{n}\left|\psi(t A) x_{i}\right|^{2} \frac{d t}{t}\right)^{1 / 2}\right\|_{\Lambda} \leqslant K_{1}\left\|\sum_{i=1}^{n} r_{i} h_{i}\right\|_{L^{1}\left(I ; \Lambda\left(H_{0}\right)\right)} .
$$

But we can write

$$
\left\|\sum_{i=1}^{n} r_{i} h_{i}\right\|_{L^{1}\left(I ; \Lambda\left(H_{0}\right)\right)}=\int_{I}\left\|\left(\int_{0}^{\infty}\left|\psi(t A) \sum_{i=1}^{n} r_{i}(s) x_{i}\right|^{2} \frac{d t}{t}\right)^{1 / 2}\right\|_{\Lambda} d s .
$$

Hence applying Lemma 5.3 to $\sum r_{i}(s) x_{i}$ for all $s$ in $I$, we obtain

$$
\left\|\sum_{i=1}^{n} r_{i} h_{i}\right\|_{L^{1}\left(I ; \Lambda\left(H_{0}\right)\right)} \leqslant C \int_{I}\left\|\sum_{i=1}^{n} r_{i}(s) x_{i}\right\|_{\Lambda} d s=C\left\|\sum_{i=1}^{n} r_{i} x_{i}\right\|_{L^{1}(I ; \Lambda)},
$$

whence the result follows by (5.1).

Proof of Theorem 5.2. Let $\alpha$ be such that $\omega<\mu<\alpha<2 \alpha-\mu<\nu<\pi$. Consider $\gamma$ in $H_{0}^{\infty}\left(\Sigma_{\mu}\right)$ such that $\gamma=\psi^{2}$ with $\psi$ in $H_{0}^{\infty}\left(\Sigma_{\mu}\right)$ and satisfying

$$
\exists K>0 \text { such that } \forall s \in \mathbb{R}, \hat{\gamma}_{e}(s) \geqslant K e^{-\alpha|s|},
$$


where $\gamma_{e} \in L^{1}(\mathbb{R})$ is defined by $\gamma_{e}(t)=\gamma\left(e^{t}\right)$. Such a $\gamma$ exists (see [8, Example 4.7]). We then obtain a constant $C>0$ such that

$$
\begin{aligned}
& \forall b \in H_{0}^{\infty}\left(\Sigma_{\nu} ; B(H)\right), \quad \exists \beta \in L^{\infty}\left(\mathbb{R}_{+} ; B(H)\right) \cap L^{1}\left(\mathbb{R}_{+}, \frac{d t}{t} ; B(H)\right) \text { such that } \\
& \|\beta\|_{L^{\infty}\left(\mathbb{R}_{+} ; B(H)\right)} \leqslant C\|b\|_{H^{\infty}\left(\Sigma_{\nu} ; B(H)\right)} \quad \text { and } \quad \forall z \in \Sigma_{\mu}, \quad b(z)=\int_{0}^{\infty} \beta(t) \gamma(t z) \frac{d t}{t} .
\end{aligned}
$$

This result is proved in the scalar case (that is, for $b \in H_{0}^{\infty}\left(\Sigma_{\nu}\right)$ ) in the course of the proof of Theorem 4.4 in [8]. It is easy to check that the same proof works as well for vector-valued functions.

In the sequel, we simply denote by $u$ the functional calculus map associated with $\mathscr{A}$ and which is defined on $H_{0}^{\infty}\left(\Sigma_{\nu} ; B(H)\right)$ by (2.1). For $b \in H_{0}^{\infty}\left(\Sigma_{\nu} ; B(H)\right)$, $\theta \in(\omega, \mu)$ and $x \in \Lambda \otimes H$, we have

$$
u(b) x=\frac{1}{2 \pi i} \int_{\Gamma_{\theta}}\left((\lambda-A)^{-1} \otimes \int_{0}^{\infty} \beta(t) \gamma(t \lambda) \frac{d t}{t}\right) x d \lambda,
$$

where $\beta$ is given by (5.5). So, by Fubini's theorem,

$$
u(b) x=\int_{0}^{\infty}(\gamma(t A) \otimes \beta(t)) x \frac{d t}{t} .
$$

Let us consider $\left(x, x^{*}\right) \in(\Lambda \otimes H) \times\left(\Lambda^{*} \otimes H\right)$. We can write $x=\sum_{i=1}^{n} x_{i} \otimes e_{i}$ and $x^{*}=\sum_{i=1}^{n} x_{i}^{*} \otimes e_{i}$, where $\left\{x_{i}\right\}_{i=1}^{n} \subset \Lambda, \quad\left\{x_{i}^{*}\right\}_{i=1}^{n} \subset \Lambda^{*}$ and $\left\{e_{i}\right\}_{i=1}^{n}$ is an orthonormal system of $H$. Then letting $\beta_{i, j}(t)=\left\langle\beta(t) e_{j}, e_{i}\right\rangle_{H}$, we have

$$
\begin{aligned}
\left\langle u(b) x, x^{*}\right\rangle & =\int_{0}^{\infty} \sum_{1 \leqslant i, j \leqslant n} \beta_{i, j}(t)\left\langle\gamma(t A) x_{j}, x_{i}^{*}\right\rangle_{\Lambda, \Lambda^{*}} \frac{d t}{t} \\
& =\int_{0}^{\infty} \sum_{1 \leqslant i, j \leqslant n} \beta_{i, j}(t)\left\langle\psi(t A) x_{j}, \psi(t A)^{*} x_{i}^{*}\right\rangle_{\Lambda, \Lambda^{*}} \frac{d t}{t} \\
& =\int_{\Omega} \int_{0}^{\infty} \sum_{1 \leqslant i, j \leqslant n} \beta_{i, j}(t)\left(\psi(t A) x_{j}\right)(\omega)\left(\psi(t A)^{*} x_{i}^{*}\right)(\omega) \frac{d t}{t} d \omega
\end{aligned}
$$

by Fubini's theorem. Hence $\left|\left\langle u(b) x, x^{*}\right\rangle\right|$ is less than

$$
\begin{aligned}
& \int_{\Omega} \int_{0}^{\infty}\|\beta(t)\|_{B(H)}\left(\sum_{i=1}^{n}\left|\left(\psi(t A) x_{i}\right)(\omega)\right|^{2}\right)^{1 / 2}\left(\sum_{i=1}^{n}\left|\left(\psi(t A)^{*} x_{i}^{*}\right)(\omega)\right|^{2}\right)^{1 / 2} \frac{d t}{t} d \omega \\
& \leqslant\|\beta\|_{L^{\infty}} \int_{\Omega}\left(\int_{0}^{\infty} \sum_{i=1}^{n}\left|\left(\psi(t A) x_{i}\right)(\omega)\right|^{2} \frac{d t}{t}\right)^{1 / 2}\left(\int_{0}^{\infty} \sum_{i=1}^{n}\left|\left(\psi(t A)^{*} x_{i}^{*}\right)(\omega)\right|^{2} \frac{d t}{t}\right)^{1 / 2} d \omega \\
& \quad \text { (by the Cauchy-Schwarz inequality) } \\
& \leqslant K\|b\|\left\|\left(\int_{0}^{\infty} \sum_{i=1}^{n}\left|\psi(t A) x_{i}\right|^{2} \frac{d t}{t}\right)^{1 / 2}\right\|\left\|\left(\int_{0}^{\infty} \sum_{i=1}^{n}\left|\psi(t A)^{*} x_{i}^{*}\right|^{2} \frac{d t}{t}\right)^{1 / 2}\right\|_{\Lambda^{*}} .
\end{aligned}
$$

The Banach lattice $\Lambda$ is reflexive because it is $B$-convex [21, Theorem 1.c.5]. 
Therefore $A^{*}$ admits a bounded $H^{\infty}\left(\Sigma_{\mu}\right)$ functional calculus on $\Lambda^{*}$. Moreover, $\psi(t A)^{*}=\tilde{\psi}\left(t A^{*}\right)$, where $\tilde{\psi} \in H_{0}^{\infty}\left(\Sigma_{\mu}\right)$ is defined by $\tilde{\psi}(z)=\overline{\psi(\bar{z})}$ for any $z \in \Sigma_{\mu}$. Hence applying Lemma 5.4 to $A$ and $A^{*}$, we obtain

$$
\begin{aligned}
\left|\left\langle u(b) x, x^{*}\right\rangle\right| & \leqslant K C^{2}\|b\|\left\|\left(\sum_{i=1}^{n}\left|x_{i}\right|^{2}\right)^{1 / 2}\right\|_{\Lambda}\left\|\left(\sum_{i=1}^{n}\left|x_{i}^{*}\right|^{2}\right)^{1 / 2}\right\|_{\Lambda^{*}} \\
& =K C^{2}\|b\|\|x\|_{\Lambda(H)}\left\|x^{*}\right\|_{\Lambda^{*}(H)} .
\end{aligned}
$$

From the $B$-convexity of $\Lambda$, it follows that $\Lambda(H)^{*}$ is canonically identified with $\Lambda^{*}(H)$. Then by the density of $\Lambda \otimes H$ in $\Lambda(H)$ and $\Lambda^{*} \otimes H$ in $\Lambda^{*}(H)$, we infer from the above estimate that $\|u(b)\| \leqslant K C^{2}\|b\|$.

In order to apply Theorem 5.2, we need to extend to $\Lambda(X)$ an operator $B$ defined on some Banach space $X$. The situation is simpler than in Proposition 5.1.

Lemma 5.5. Let $B$ be a pseudosectorial operator of type $\omega^{\prime}$ on $X$ and let $\mu^{\prime} \in\left(\omega^{\prime}, \pi\right)$.

(i) The operator $I_{\Lambda} \otimes B$, defined on $\Lambda \otimes D(B)$, is closable and its closure $\mathscr{B}$ is pseudosectorial of type $\omega^{\prime}$. Moreover, $D(\mathscr{B})=\Lambda(D(B))$, where $D(B)$ is equipped with its graph norm $\|x\|_{D(B)}=\|x\|_{X}+\|B x\|_{X}$.

(ii) If $B$ is sectorial of type $\omega^{\prime}$, so is $\mathscr{B}$.

(iii) If $B$ admits a bounded $H^{\infty}\left(\Sigma_{\mu^{\prime}}\right)$ functional calculus, so does $\mathscr{B}$.

Proof. Let $\left(x_{n}\right)_{n=1}^{\infty}$ be a sequence in $\Lambda \otimes D(B)$ such that $x_{n} \rightarrow 0$ and $\left(I_{\Lambda} \otimes B\right) x_{n} \rightarrow y$. Then there is a subsequence $\left(x_{n}^{\prime}\right)_{n=1}^{\infty}$ of $\left(x_{n}\right)_{n=1}^{\infty}$ such that for almost all $\omega$ in $\Omega, x_{n}^{\prime}(\omega) \rightarrow 0$ and $B\left(x_{n}^{\prime}(\omega)\right) \rightarrow y(\omega)$. Since $B$ is closed, we have $y=0$ and therefore $I_{\Lambda} \otimes B$ is closable. Then our description of $D(\mathscr{B})$ is a straightforward consequence of the definition of $\Lambda(D(B))$. The operator $\mathscr{B}$ is pseudosectorial on account of the following fact:

$$
\rho(B) \subset \rho(\mathscr{B}) \quad \text { and } \quad \forall \lambda \in \rho(B), \quad(\lambda-\mathscr{B})^{-1}=\overline{I_{\Lambda} \otimes(\lambda-B)^{-1}} .
$$

The assertion (ii) is then clear. From (5.6), it follows that for any $f$ in $H_{0}^{\infty}\left(\Sigma_{\mu^{\prime}}\right)$, $f(\mathscr{B})=\overline{I_{\Lambda} \otimes f(B)}$, which yields (iii).

Then, as an application of Theorem 5.2 and Proposition 2.6, we have the following.

Corollary 5.6. Let $A$ be an operator on a B-convex Banach lattice $\Lambda$ admitting a bounded $H^{\infty}\left(\Sigma_{\mu}\right)$ functional calculus and let $B$ be a pseudosectorial operator of type $\omega^{\prime}$ on a Hilbert space $H$. Denote by $\mathscr{A}$ and $\mathscr{B}$ the extensions described above of $A$ and $B$ to $\Lambda(H)$ and assume that $\mu+\omega^{\prime}<\pi$.

Then $\mathscr{A}+\mathscr{B}$ is a closed one-to-one operator on $\Lambda(H)$ with dense range and

$$
\exists C>0 \text { such that } \forall x \in D(\mathscr{A}) \cap D(\mathscr{B}),\|\mathscr{A} x\| \leqslant C\|(\mathscr{A}+\mathscr{B}) x\| .
$$

Proof of Theorem 1.4. Under the assumptions of Theorem 1.4, the fact that $B \otimes I_{H}$ and $I_{L^{q}} \otimes C$ are closable follows from Proposition 5.1 and Lemma 5.5. We let $\tilde{B}=\overline{B \otimes I_{H}}$ and $\tilde{C}=\overline{I_{L^{q}} \otimes C}$. Then by Corollary $5.6, \tilde{B}+\tilde{C}$ is a closed 
one-to-one operator with a dense range. The fact that $\tilde{B}+\tilde{C}$ is actually sectorial of type less than $\frac{1}{2} \pi$ can be easily deduced from Theorem 5.2 but also follows from the estimates given in $[9, \S 3]$.

Now let $T>0$ and $1<p<\infty$. Consider $A=d / d t$ on $L^{p}(0, T)$ with domain $W_{0}^{1, p}(0, T)$ and let

$$
\Lambda=L^{p}\left(0, T ; L^{q}\left(\Omega_{1}\right)\right) .
$$

Then $A \otimes I_{L^{q}\left(\Omega_{1}\right)}$ is closable in $\Lambda$ and $\overline{A \otimes I_{L^{q}\left(\Omega_{1}\right)}}$ admits a bounded $H^{\infty}\left(\Sigma_{\eta}\right)$ functional calculus for any $\eta>\frac{1}{2} \pi$ (see [11]). The space $\Lambda$ is a $B$-convex Banach lattice and it is easy to check that $\Lambda(H)$ can be identified with $L^{p}\left(0, T ; L^{q}\left(\Omega_{1} ; H\right)\right)$ and that the closure of $\overline{A \otimes I_{L^{q}\left(\Omega_{1}\right)}} \otimes I_{H}$, provided by Proposition 5.1, is $\mathscr{A}=d / d t$ on $L^{p}\left(0, T ; L^{q}\left(\Omega_{1} ; H\right)\right)$ with domain $W_{0}^{1, p}\left(0, T ; L^{q}\left(\Omega_{1} ; H\right)\right)$.

We denote by $\mathscr{B}$ and $\mathscr{C}$, respectively, the closures of $I_{L^{p}(0, T)} \otimes \tilde{B}$ and $I_{L^{p}(0, T)} \otimes \tilde{C}$ in $\Lambda(H)$. On the other hand, by Lemma $5.5, I_{L^{p}(0, T)} \otimes B$ is closable on $\Lambda$ and its closure admits a bounded $H^{\infty}\left(\Sigma_{\mu}\right)$ functional calculus. It can easily be checked that $\mathscr{B}$ is the closure of $\overline{I_{L^{p}(0, T)} \otimes B} \otimes I_{H}$. We also have that $\mathscr{C}$ is the closure of $I_{\Lambda} \otimes C$ and is a sectorial operator of type $\omega^{\prime}$ on $\Lambda(H)$.

By Theorem 3.2, $\left(\overline{A \otimes I_{L^{q}\left(\Omega_{1}\right)}}, \overline{I_{L^{p}(0, T)} \otimes B}\right)$ admits on $\Lambda$ a bounded $H^{\infty}\left(\Sigma_{\alpha} \times \Sigma_{\beta}\right)$ joint functional calculus, for any $(\alpha, \beta)$ in $\left(\frac{1}{2} \pi, \pi\right) \times(\mu, \pi)$ and, by Proposition 5.1, so does $(\mathscr{A}, \mathscr{B})$. By Proposition 2.7, this implies that there exists $C_{1}>0$ such that

$$
\forall x \in D(\mathscr{A}) \cap D(\mathscr{B}), \quad\|\mathscr{A} x\| \leqslant C_{1}\|(\mathscr{A}+\mathscr{B}) x\| \text {. }
$$

Now let $S=\overline{A \otimes I_{L^{q}}\left(\Omega_{1}\right)}+\overline{I_{L^{p}(0, T)} \otimes B}$. Then by Corollary 4.3, $S$ admits a bounded $H^{\infty}\left(\Sigma_{\nu}\right)$ functional calculus, for any $\nu>\frac{1}{2} \pi$. It is standard to verify that $\overline{S \otimes I_{H}}=\mathscr{A}+\mathscr{B}$. Then, in view of Corollary 5.6, there exists $C_{2}>0$ such that

$$
\forall x \in D(\mathscr{A}) \cap D(\mathscr{B}) \cap D(\mathscr{C}), \quad\|(\mathscr{A}+\mathscr{B}) x\| \leqslant C_{2}\|(\mathscr{A}+\mathscr{B}+\mathscr{C}) x\| .
$$

From (5.8) and (5.9), it follows of course that $\mathscr{A}(\mathscr{A}+\mathscr{B}+\mathscr{C})^{-1}$ is bounded. As is well known, this is equivalent to the maximal regularity of $\tilde{B}+\tilde{C}$.

\section{A characterization of Hilbert spaces in terms of functional calculi}

Let $X$ be a Banach space, $p \in(1,+\infty)$ and let $\mathbb{T}=\{z \in \mathbb{C}:|z|=1\}$. We will start with the study of the first derivation operator on $L^{p}(\mathbb{T} ; X)$. So consider $A=i z(d / d z)$ on $L^{p}(\mathbb{T})$ with domain $D(A)=W^{1, p}(\mathbb{T})$, and $\mathscr{A}=i z(d / d z)$ on $L^{p}(\mathbb{T} ; X)$ with domain $D(\mathscr{A})=W^{1, p}(\mathbb{T} ; X)$ which is also the closure of $A \otimes I_{X}$ in $L^{p}(\mathbb{T} ; X)$. It is known that $\mathscr{A}$ is pseudosectorial of type $\frac{1}{2} \pi$ and that, for any $\mu$ in $\left(\frac{1}{2} \pi, \pi\right), \mathscr{A}$ admits a bounded $H_{0}^{\infty}\left(\Sigma_{\mu}\right)$ functional calculus if and only if $X$ is a UMD space $[\mathbf{1 1}, \mathbf{2 9}]$.

We recall (see $\S 5$ ), that $B(X)$ is canonically identified with a closed subalgebra of $E_{\mathscr{A}}$. Then we have the following characterization.

TheOREM 6.1. Let $\frac{1}{2} \pi<\mu<\pi$. The operator $\mathscr{A}$ admits a bounded $H_{0}^{\infty}\left(\Sigma_{\mu} ; B(X)\right)$ functional calculus if and only if $X$ is isomorphic to a Hilbert space.

Proof. The 'if' part is a consequence of Theorem 5.2. So let us assume that $\mathscr{A}$ admits a bounded $H_{0}^{\infty}\left(\Sigma_{\mu} ; B(X)\right)$ functional calculus. We denote as usual by

$$
u_{\mathscr{A}}: H_{0}^{\infty}\left(\Sigma_{\mu} ; B(X)\right) \rightarrow B\left(L^{p}(\mathbb{T} ; X)\right)
$$

the induced bounded homomorphism. Then we will show that $X$ is of type 2 and 
of cotype 2 (see Definition 2.8). This, by Kwapien's theorem [18], will yield the conclusion.

Let us prove first that $X$ is of type 2. So let $\left\{x_{k}\right\}_{k=1}^{n}$ be a finite subset of $X$ and fix $\left(e, e^{*}\right)$ in $X \times X^{*}$ with $\left\langle e, e^{*}\right\rangle=\|e\|_{X}=\left\|e^{*}\right\|_{X^{*}}=1$.

The sequence $\left(\sqrt{2^{k}} e^{i(\pi / 4)}\right)_{k \geqslant 1}$ satisfies the condition (i) of Theorem 2.9 (the verification is left to the reader). Then using the transformation $z \mapsto z^{2}$, we obtain a sequence $\left(f_{k}\right)_{k \geqslant 1}$ in $H^{\infty}\left(\Sigma_{\pi}\right)$ and a constant $M>0$ such that

$$
\forall k \geqslant 1, f_{k}\left(i 2^{k}\right)=1 ; \quad \forall j \neq k, f_{j}\left(i 2^{k}\right)=0 ; \quad \text { and } \quad \forall z \in \Sigma_{\pi}, \sum_{k \geqslant 1}\left|f_{k}(z)\right| \leqslant M .
$$

Now, consider, for $1 \leqslant k \leqslant n, T_{k} \in B(X)$ defined by $T_{k} x=e^{*}(x)\left(x_{k} /\left\|x_{k}\right\|\right)$ for all $x \in X$ (we may assume $x_{k} \neq 0$ ). For $z$ in $\Sigma_{\pi}$, define $\left.f(z)=\sum_{k}\right\rangle_{1} f_{k}(z) T_{k}$. Then $f \in H^{\infty}\left(\Sigma_{\pi} ; B(X)\right),\|f\|_{H^{\infty}\left(\Sigma_{\pi} ; B(X)\right)} \leqslant M$, and for all $k \in\{1, \ldots, n\}, f\left(i 2^{k}\right)=T_{k}$.

We also consider $P$ in $L^{p}(\mathbb{T} ; X)$ defined as follows: for all $z \in \mathbb{T}, P(z)=$ $\left(\sum_{k=1}^{n}\left\|x_{k}\right\| z^{2^{k}}\right) e$.

Let $\varepsilon_{k}$ be defined on $\mathbb{T}$ by $\varepsilon_{k}(z)=z^{2^{k}}$. It is known (see [26]) that there is a constant $C>0$ such that, for any $y_{1}, \ldots, y_{n}$ in a Banach space $Y$,

$$
C^{-1}\left\|\sum_{k=1}^{n} \varepsilon_{k} y_{k}\right\|_{L^{p}(\mathbb{T} ; Y)} \leqslant\left\|\sum_{k=1}^{n} r_{k} y_{k}\right\|_{L^{1}(I ; Y)} \leqslant C\left\|\sum_{k=1}^{n} \varepsilon_{k} y_{k}\right\|_{L^{p}(\mathbb{T} ; Y)} .
$$

In particular, this yields (with $Y=\mathbb{C}$ )

$$
\|P\|_{L^{p}(\mathbb{T} ; X)} \leqslant C\left(\sum_{k=1}^{n}\left\|x_{k}\right\|^{2}\right)^{1 / 2} .
$$

Now, we need to introduce the auxiliary functions defined, for $N$ in $\mathbb{N}$ and $\lambda \in \Sigma_{\mu}$, by

$$
\xi_{N}(\lambda)=\frac{1}{1+\lambda / N}-\frac{1}{1+\lambda N}
$$

For any $N>0, \xi_{N} \in H_{0}^{\infty}\left(\Sigma_{\mu}\right)$ and $\left\|\xi_{N}\right\|_{H^{\infty}\left(\Sigma_{\mu}\right)} \leqslant C_{\mu}$, where $C_{\mu}$ does not depend on $N$. Using the Cauchy Residue Theorem, one obtains

$$
\forall z \in \mathbb{T}, \quad\left(u_{\mathscr{A}}\left(\xi_{N} f\right) P\right)(z)=\sum_{k=1}^{n} \xi_{N}\left(i 2^{k}\right) z^{2^{k}} x_{k}
$$

This, combined with (6.1) and (6.2), implies that

$$
\left\|\sum_{k=1}^{n} r_{k} \xi_{N}\left(i 2^{k}\right) x_{k}\right\|_{L^{1}(I ; X)} \leqslant C^{2} C_{\mu} M\left\|u_{\mathscr{A}}\right\|\left(\sum_{k=1}^{n}\left\|x_{k}\right\|^{2}\right)^{1 / 2} .
$$

Finally, for any $z$ in $\Sigma_{\mu}, \lim _{N \rightarrow+\infty} \xi_{N}(z)=1$. Thus, passing to the limit in (6.3), we see that $X$ is of type 2 .

In order to prove that $X$ is of cotype 2, we follow the same steps, after exchanging the $T_{k}$ with the operators defined by, for all $x \in X, S_{k} x=x_{k}^{*}(x) e$, where $x_{k}^{*} \in X^{*},\left\|x_{k}^{*}\right\|_{X^{*}}=1$ and $x_{k}^{*}\left(x_{k}\right)=\left\|x_{k}\right\|$.

We will now show how to use transference methods in order to obtain the same characterization if we replace $\mathbb{T}$ by $\mathbb{R}$. So let $X$ be a Banach space, $1<p<+\infty$, 
$A_{0}=d / d t$ on $L^{p}(\mathbb{R})$ with domain $W^{1, p}(\mathbb{R})$, and $\mathscr{A}_{0}=d / d t$ on $L^{p}(\mathbb{R} ; X)$ with domain $W^{1, p}(\mathbb{R} ; X)$. We have a similar result.

THeOREM 6.2. Let $\frac{1}{2} \pi<\mu<\pi$. The operator $\mathscr{A}_{0}$ admits a bounded $H^{\infty}\left(\Sigma_{\mu} ; B(X)\right)$ functional calculus if and only if $X$ is isomorphic to a Hilbert space.

Proof. Notice first that $-\mathscr{A}_{0}$ and $-\mathscr{A}$ generate groups of translations in $L^{p}(\mathbb{R} ; X)$ and $L^{p}(\mathbb{T} ; X)$, which we denote by $\left(e^{-t \mathscr{A}_{0}}\right)_{t \in \mathbb{R}}$ and $\left(e^{-t \mathscr{A}}\right)_{t \in \mathbb{R}}$.

Lemma 6.3. For any $k$ in $L^{1}(\mathbb{R} ; B(X))$,

$$
\left\|\int_{\mathbb{R}} e^{-t \mathscr{A}} k(t) d t\right\|_{B\left(L^{p}(\mathbb{T} ; X)\right)} \leqslant\left\|\int_{\mathbb{R}} e^{-t \mathscr{A}_{0}} k(t) d t\right\|_{B\left(L^{p}(\mathbb{R} ; X)\right)} .
$$

In the case when $k$ belongs to $L^{1}(\mathbb{R})$, this result follows from some well-known work of Calderon on transference, using the group action of $\mathbb{R}$ on $\mathbb{T}$ by translation (see, for example, [7, Chapters 1 and 2]). This extension to the $B(X)$-valued case is straightforward and left to the reader.

Suppose now that $u_{\mathscr{A}_{0}}: H_{0}^{\infty}\left(\Sigma_{\mu} ; B(X)\right) \rightarrow B\left(L^{p}(\mathbb{R}, X)\right)$ is bounded. Let $\mathscr{R}_{0}$ be the algebra of all rational functions, belonging to $H_{0}^{\infty}\left(\Sigma_{\mu}\right)$, with poles outside $\overline{\Sigma_{\mu}}$. Clearly $\mathscr{R}_{0}$ is a subalgebra of $H_{0}^{\infty}\left(\Sigma_{\mu}\right)$. Any $f$ in $\mathscr{R}_{0}$ is the Laplace transform of some $k$ in $L^{1}\left(\mathbb{R}_{+}\right)$. More precisely,

$$
\forall z \in \Sigma_{\pi / 2}, \quad f(z)=\int_{0}^{\infty} e^{-t z} k(t) d t .
$$

This inspired the so-called Phillips functional calculus and it is known that for any $f$ in $\mathscr{R}_{0}, u_{\mathscr{A}_{0}}(f)$ is equal to $\int_{0}^{\infty} e^{-t \mathscr{A}_{0}} k(t) d t$, where $k$ is given by (6.4). This can be extended in the following way. For any $f$ in $\mathscr{R}_{0} \otimes B(X)$, there exists $k$ in $L^{1}\left(\mathbb{R}_{+} ; B(X)\right)$ such that

$$
\forall z \in \Sigma_{\pi / 2}, \quad f(z)=\int_{0}^{\infty} e^{-t z} k(t) d t \quad \text { and } \quad u_{\mathscr{A}_{0}}(f)=\int_{0}^{\infty} e^{-t \mathscr{A}_{0}} k(t) d t .
$$

This is also true for $u_{\mathscr{A}}$. So, by Lemma $6.3, u_{\mathscr{A}}$ is bounded on $\mathscr{R}_{0} \otimes B(X)$ and

$$
\exists K>0 \text { such that } \forall f \in \mathscr{R}_{0} \otimes B(X),\left\|u_{\mathscr{A}}(f)\right\| \leqslant K\|f\|_{H^{\infty}\left(\Sigma_{\mu} ; B(X)\right)} .
$$

Now consider $f$ in $H_{0}^{\infty}\left(\Sigma_{\mu}\right) \otimes B(X)$. For $\varepsilon>0$ and $z$ in $\mathbb{C} \backslash\{-(1 / \varepsilon)\}$, we set $g_{\varepsilon}(z)=(\varepsilon+z) /(1+\varepsilon z)$. Notice that $g_{\varepsilon}\left(\overline{\Sigma_{\mu}}\right)$ is a compact subset of $\Sigma_{\mu}$. Hence it is not hard to deduce from Runge's theorem that there is a sequence $\left(f_{n}\right)_{n \geqslant 1}$ in $\mathscr{R}_{0} \otimes B(X)$ such that $f_{n} \circ g_{\varepsilon}$ converges to $f \circ g_{\varepsilon}$ uniformly on $\Sigma_{\mu}$. Now fix $m \in \mathbb{N}$. It follows from (6.5) that

$$
\begin{aligned}
& \forall \varepsilon>0, \quad \forall m>0, \quad \forall n>0, \\
& \quad\left\|u_{\mathscr{A}}\left(\varphi_{m}\left(f_{n} \circ g_{\varepsilon}\right)\right)\right\| \leqslant K\left\|\varphi_{m}\right\|_{H^{\infty}\left(\Sigma_{\mu}\right)}\left\|f_{n} \circ g_{\varepsilon}\right\|_{H^{\infty}\left(\Sigma_{\mu} ; B(X)\right)} .
\end{aligned}
$$

Since $\left(\varphi_{m}\right)_{m \geqslant 1}$ is uniformly bounded in $H^{\infty}\left(\Sigma_{\mu}\right)$, there is a constant $K^{\prime}>0$ such that

$$
\forall \varepsilon>0, \quad \forall m>0, \quad\left\|u_{\mathscr{A}}\left(\varphi_{m}\left(f \circ g_{\varepsilon}\right)\right)\right\| \leqslant K^{\prime}\|f\|_{H^{\infty}\left(\Sigma_{\mu} ; B(X)\right)} .
$$


By Lebesgue's dominated convergence theorem, we have

$$
\forall x \in X, \quad \lim _{\varepsilon \rightarrow 0} u_{\mathscr{A}}\left(\varphi_{m}\left(f \circ g_{\varepsilon}\right)\right) x=u_{\mathscr{A}}(f) \varphi_{m}(A) x .
$$

Therefore, letting $m$ tend to $+\infty$ we obtain $\left\|u_{\mathscr{A}}(f)\right\| \leqslant K^{\prime}\|f\|_{H^{\infty}\left(\Sigma_{\mu} ; B(X)\right)}$. Finally, notice that it follows from the proof of Theorem 6.1 that the boundedness of $u_{\mathscr{A}}$ restricted to $H_{0}^{\infty}\left(\Sigma_{\mu}\right) \otimes B(X)$ actually implies that $X$ is isomorphic to a Hilbert space.

REMARK 6.4. Let $1<p<\infty$ and let $A_{0}=d / d t$ on $L^{p}(\mathbb{R})$ as above. The classical proof of the fact that for any $\mu>\frac{1}{2} \pi, A_{0}$ admits a bounded $H^{\infty}\left(\Sigma_{\mu}\right)$ functional calculus relies on Mihlin's multiplier theorem on $L^{p}(\mathbb{R})$. The arguments involved in this proof can be adapted to the operator-valued framework; hence it is possible to derive a direct proof of Theorem 5.2 for $A_{0}$ from Mihlin's theorem on $L^{p}(\mathbb{R} ; H)$ for $B(H)$-valued multipliers [2, Theorem 6.1.6]. In the same manner, if we assume that for a given Banach space $X$, Mihlin's theorem holds on $L^{p}(\mathbb{R} ; X)$ for $B(X)$-valued multipliers, then one can show that $\mathscr{A}_{0}$ admits a bounded $H^{\infty}\left(\Sigma_{\mu} ; B(X)\right)$ functional calculus on $L^{p}(\mathbb{R} ; X)$ for any $\mu>\frac{1}{2} \pi$. Thus as a consequence of Theorem 6.2, we obtain that $X$ is necessarily isomorphic to Hilbert space. The fact that the operator-valued Mihlin multiplier theorem only holds on Hilbert spaces has been known for a long time and goes back to $G$. Pisier (unpublished).

REMARK 6.5. Given a sectorial operator $A$ which admits a bounded $H^{\infty}\left(\Sigma_{\mu}\right)$ functional calculus on a Banach space $X$, a natural question is: does $A$ automatically admit a bounded $H^{\infty}\left(\Sigma_{\mu} ; E_{A}\right)$ functional calculus? This holds true when $X$ is a Hilbert space. This result is implicit in [19] and its proof relies upon techniques from [23]. We were informed by Albrecht and McIntosh that they have also obtained this result (paper in preparation). When we leave Hilbert spaces, the situation turns out to be much more complicated. Indeed, Theorem 6.2 shows that on $L^{p}$-spaces $(1<p<\infty, p \neq 2)$, the boundedness of $H^{\infty}\left(\Sigma_{\mu}\right)$ functional calculus for an operator $A$ does not imply the boundedness of $H^{\infty}\left(\Sigma_{\mu} ; E_{A}\right)$ functional calculus.

\section{References}

1. D. Albrecht, 'Functional calculi of commuting unbounded operators', Ph.D. thesis, Monash University, Australia, 1994.

2. J. BERGH and A. J. LöFströM, Interpolation spaces (Springer, New York, 1976).

3. J. Bourgain, 'Some remarks on Banach spaces in which martingale difference sequences are unconditional', Ark. Mat. 21 (1983) 163-168.

4. J. Bourgain, 'New Banach space properties of the disk algebra and $H^{\infty}$, Acta Math. 152 (1984) $1-48$.

5. J. Bourgain, 'Vector valued singular integrals and the $H^{1}$-BMO duality', Probability theory and harmonic analysis (eds J.-A. Chao and W. A. Woyczynski, Dekker, New York, 1986), pp. 1-19.

6. J. Bourgain and W. DAvis, 'Martingale transforms and complex uniform convexity', Trans. Amer. Math. Soc. 294 (1986) 501-515.

7. R. R. CoIfMAN and G. WeIss, Transference methods in analysis, CBMS Regional Conference Series in Mathematics 31 (American Mathematical Society, Providence, R.I., 1977).

8. M. Cowling, I. Doust, A. McIntosh and A. YAgi, 'Banach space operators with a bounded $H^{\infty}$ functional calculus', J. Austral. Math. Soc. Ser. A 60 (1996) 51-89.

9. G. DA Prato and P. Grisvard, 'Sommes d'opérateurs linéaires et équations différentielles opérationnelles', J. Math. Pures Appl. 54 (1975) 305-387.

10. L. DE SimON, 'Un' applicazione della theoria degli integrali singolari allo studio delle 
equazioni differenziali lineare astratte del primo ordine', Rend. Sem. Mat. Univ. Padova (1964) 205-223.

11. G. Dore and A. Venni, 'On the closedness of the sum of two closed operators', Math. Z. 196 (1987) 189-201.

12. G. Dore and A. Venni, 'Some results about complex powers of closed operators', J. Math. Anal. Appl. 149 (1990) 124-136.

13. T. Figiel, W. Johnson and L. Tzafriri, 'On Banach lattices and spaces having local unconditional structure, with applications to Lorentz function spaces', J. Approx. Theory 13 (1975) 395-412.

14. E. Franks and A. McIntosh, 'Discrete quadratic estimates and holomorphic functional calculi of operators in Banach spaces', Macquarie Mathematics Report 95/192, 1995.

15. J. B. GarnetT, Bounded analytic functions (Academic Press, New York, 1981).

16. J. A. Goldstein, Semigroups of linear operators and applications (Oxford University Press, New York, 1985).

17. J. L. Krivine, Théorèmes de factorisation dans les espaces de Banach réticulés, Séminaire Maurey-Schwarz (1973/74) 22-23 (École Polytechnique, Paris, 1974).

18. S. KWAPIEN, 'Isomorphic characterizations of inner product spaces by orthogonal series with vector valued coefficients', Studia Math. 44 (1972) 583-595.

19. C. LE Merdy, 'On dilation theory for $C_{0}$-semigroups on Hilbert space', Indiana Univ. Math. J. 45 (1996) 945-959.

20. J. Lindenstrauss and L. Tzafriri, Classical Banach spaces I (Springer, Berlin, 1977).

21. J. Lindenstrauss and L. Tzafriri, Classical Banach spaces II (Springer, Berlin, 1979).

22. B. Maurey, Type et cotype dans les espaces munis de structures locales inconditionnelles, Séminaire Maurey-Schwartz (1973/74) 24-25 (École Polytechnique, Paris, 1974).

23. A. McIntosh, 'Operators which have an $H^{\infty}$ functional calculus', Miniconference on Operator Theory and Partial Differential Equations, Proceedings of the Centre for Mathematical Analysis 14 (Australian National University, Canberra, 1986), pp. 210-231.

24. A. McIntosh and A. YAgI, 'Operators of type $\omega$ without a bounded $H^{\infty}$ functional calculus', Miniconference on Operators in Analysis, Proceedings of the Centre for Mathematical Analysis 24 (Australian National University, Canberra, 1989), pp. 159-172.

25. G. Pisier, 'Some results on Banach spaces without local unconditional structures', Compositio Math. 37 (1978) 3-19.

26. G. Pisier, Les inégalités de Khintchine-Kahane d'après C. Borell, Séminaire sur la géométrie des espaces de Banach (1977/78) 7 (École Polytechnique, Paris, 1978).

27. G. Pisier, 'Quotients of Banach spaces of cotype q', Proc. Amer. Math. Soc. 85 (1982) 32-36.

28. G. PISIER, Factorization of linear operators and geometry of Banach spaces, CBMS Regional Conference Series in Mathematics 60 (American Mathematical Society, Providence, R.I., 1986).

29. J. Prüss, Evolutionary integral equations and applications, Monographs in Mathematics 87 (Birkhäuser, Basel, 1993).

30. J. Prüss and H. SoHr, 'On operators with bounded imaginary powers in Banach spaces', Math. Z. 203 (1990) 429-452.

Equipe de Mathématiques

URA CNRS 741

Université de Franche-Comté

F-25030 Besançon Cedex

France

E-mail: lancien@math.univ-fcomte.fr lemerdy@math.univ-fcomte.fr 